\title{
The effect of base column on vortex-induced vibration of a circular cylinder with low aspect ratio
}

\author{
Yuanchuan Liư ${ }^{1}$, Fushun Liu ${ }^{12}$, Enhao Wang ${ }^{3}$, Qing Xiao ${ }^{4}$, Liang $\mathrm{Li}^{4}$ \\ ${ }^{1}$ College of Engineering, Ocean University of China, Qingdao, 266100, China \\ ${ }^{2}$ Cooperative Innovation Center of Engineering Construction and Safety in \\ Shandong Blue Economic Zone, Qingdao University of Technology, Qingdao, \\ 266033, China \\ ${ }^{3}$ State Key Laboratory of Hydraulic Engineering Simulation and Safety, \\ Tianjin University, Tianjin, 300072, China \\ ${ }^{4}$ Department of Naval Architecture, Ocean and Marine Engineering, \\ University of Strathclyde, Glasgow, G4 OLZ, UK \\ *Corresponding author: percyliu@ouc.edu.cn
}

\begin{abstract}
The end condition of a cylinder is known to influence its vortex-induced vibration (VIV) response. The design of a short circular cylinder with a concentric base column attached to its bottom is adopted by some floating structures for improved stability, but how the base column would affect the VIV of the cylinder has been rarely studied. In this paper, the VIV of an elastically mounted rigid cylinder with a base column and a low aspect ratio of 2 is investigated numerically by solving the Navier-Stokes equations. The numerical methods are validated against two existing VIV studies, including a 2D cylinder and a cylinder with a finite length. The impacts of the base column on the cylinder are analysed. It is found that although the free end effects associated with fluid flowing around the cylinder end are still present, attaching the base column leads to the expansion of the lock-in regime of the cylinder response. A relationship between nondimensional response amplitude and lift coefficient is established, which takes into consideration the geometrical properties of the base column. By analysing the energy transfer from fluid to structure, the base column is also found to have significant damping effects on the cylinder response.
\end{abstract}

Keywords: Vortex-induced vibration; base column; free end effects; damping effects. 


\section{Introduction}

Vortex-induced vibration (VIV) of elastically mounted rigid cylinders has been extensively studied over the past few decades due to its significance in various fields of engineering. For a cylinder subjected to incident flow, periodic vortex shedding occurs, resulting in the oscillation of the cylinder in the transverse or crossflow direction. Under certain circumstances, the lock-in phenomenon characterised by large transverse response amplitude appears, which may lead to structural fatigue and stability issues. Comprehensive reviews of VIV in various aspects can be found in the publications of Bearman (1984), Sarpkaya (2004), Williamson and Govardhan (2004) and Williamson and Govardhan (2008).

The majority of previous VIV studies have focused on cylinders with a high aspect ratio, for which the length of the cylinder $L$ is significantly larger than its diameter $D$, e.g. $L / D=10$. In these studies, the effects of free ends were usually not taken into consideration. A thin endplate with a diameter several times that of the tested cylinder was often installed to the free end of the cylinder in VIV experiments to prevent fluid from flowing around the end (Hover et al., 2004; Morse et al., 2008; Bourdier and Chaplin, 2012). For numerical investigations at low Reynolds numbers of $R e<200$, as flow is intrinsically laminar, two-dimensional (2D) Computational Fluid Dynamics (CFD) simulations were typically carried out (Bao et al., 2011; Zhao, 2013; Wang et al., 2019). As the Reynolds number $R e$ increases, although a fully three-dimensional (3D) domain was set up, the simulated cylinder directly pierced through the two domain boundaries normal to it and a periodic or symmetry boundary condition was applied to them, without modelling the free ends (Zhao et al., 2014; Wang et al., 2017; Navrose and Mittal, 2019).

However, existing researches on the flow past stationary cylinders with low aspect ratios demonstrated that the presence of the free end has significant impacts on the vortex formation and wake of the cylinder (Palau-Salvador et al., 2009; Sumner, 2013; Fukuoka et al., 2016). In their experimental study of the flow around cylinders with aspect ratios of $0.1 \leq L / D \leq 2$, Gonçalves et al. (2015) observed two recirculation regions with one formed over the free end and the other behind the cylinder, and 
concluded that the von Kármán vortex street typically found for cylinders with high $L / D$ diminished as the aspect ratio decreased. Benitz et al. (2016) performed a series of CFD simulations for a circular cylinder across a wide range of aspect ratios from 1 to 19 and found that the tip vortices associated with the free end dominated the flow at aspect ratios smaller than 3, with no vortex shedding from the cylinder surface.

The flow results for stationary finite-length cylinders suggest that the VIV of a cylinder with a free end can be different from that with a high $L / D$, as has been pointed out by some researchers. Kitagawa et al. (1999) investigated the VIV of a pivoted cylinder with a free end via wind tunnel experiments and observed the so-called endcell-induced vibration (ECIV) at a wind speed higher than the threshold for VIV. They attributed ECIV to the vortex shedding associated with the free end. Morse et al. (2008) experimentally studied the VIV of a rigid cylinder with and without an endplate attached to its free end at $L / D=8$. Comparisons between the results from the two different conditions revealed that the response amplitude was considerably higher in the lower branch and there was no clear transition between the upper and lower branches when the endplate was removed. Zhao and Cheng (2014) analysed the effects of the free end on the response of a cylinder of a finite length by conducting CFD simulations with $L / D$ varying from 1 to 20 at $R e=300$. The vortices generated from the free end was found to affect the VIV response amplitude and frequency of the cylinder even at a high $L / D$ of 10 . Kinaci et al. (2016) argued that the flow around the tip or free end of the cylinder could not be eliminated completely in VIV experiments and proposed a tip-flow correction factor to represent the lift loss caused by the vortices associated with the free end. Gonçalves et al. (2018) carried out VIV experiments for cylinders with very low aspect ratios of $0.2 \leq L / D \leq 2$ and concluded that the vortex shedding around the free end was the dominant source for the resonant behaviour of the cylinder with a $L / D$ lower than 0.5. Azadeh-Ranjbar et al. (2018) exposed both ends of finite-length rigid circular cylinders to the flow in their wind tunnel experiments and spotted a broadened lock-in regime by about $200 \%$ as well as a $230 \%$ increase in the peak response amplitude as $L / D$ was decreased from 28.8 to 5 .

It can then be summarised from the above literature review that the condition of the end of a cylinder plays an important role in its VIV response, especially for a 
cylinder with a low $L / D$. In the present study, the VIV of an elastically mounted circular cylinder with a different end condition, i.e. with a base column attached to its free end as illustrated in Figure 1, is investigated numerically. The cylinder and the base column are concentric but differ in diameter. It is conjectured that adding a base column to a cylinder with a free end may influence the fluid flow around the structure and consequently affect the response of the cylinder. Therefore, the objective of this work is to study the impacts of the base column on the flow past the cylinder and its VIV response. To the best of the authors' knowledge, the VIV of a cylinder with such end condition has been rarely analysed. Meanwhile, due to its advantages in stability and heave damping, the cylinder-base model is found to be adopted in some floating structure designs, e.g. the DeepCwind semi-submersible platform (Robertson et al., 2014) developed to support the OC4 floating offshore wind turbine (FOWT) as displayed in Figure 2. It is thus expected that the present study will help improve the understanding of the responses of an FOWT due to vortex shedding in current, which is more commonly referred to as vortex-induced motion (VIM) in the field of offshore engineering (Zhao et al., 2018), and provide guidance to the design of FOWTs.

The rest of this paper is organised as follows. The geometry of the structure and the flow condition analysed in the present study are firstly described in Section 2, followed by a brief introduction to the adopted numerical methods in Section 3. Two numerical tests are then carried out in Section 4 to validate the numerical tool in VIV studies and Section 5 presents the computational model established for the cylinder with a base column. Subsequently, in Section 6 the numerical results obtained for the cylinder under stationary and oscillating conditions are presented and analysed in detail. Finally, some conclusions are drawn in Section 7.

\section{Problem description}

The geometry of the cylinder with a base column studied in this work is illustrated in Figure 1. Using the OC4 DeepCwind semi-submersible platform (Robertson et al., 2014) as a reference, a relatively short cylinder with a low aspect ratio of $L / D=2$ is employed, where $L$ is the overall length of the cylinder and $D$ is its characteristic diameter. The base column has a diameter of $D_{B}=2 D$ and a length of $L_{B}=0.2 L$. A 
low mass ratio of $m^{*}=2$ is adopted, which is defined as $m^{*}=m / m_{d}$, where $m$ is the mass of the structure and $m_{d}$ is the displaced fluid mass calculated via $m_{d}=\rho \pi\left(D^{2} L_{C}+D_{B}^{2} L_{B}\right) / 4$ with $\rho$ being fluid density. The aspect ratio $L / D$ and mass ratio $m^{*}$ utilised in this study are the same as those of a finite cylinder without the base column previously investigated by Zhao and Cheng (2014), so that the effects of the base column can be assessed by making comparisons between the numerical results obtained from the two different geometries.

The cylinder is subjected to uniform flow with a flow speed of $U$ and is only allowed to oscillate in the transverse direction. The Reynolds number is defined as $R e=U D / v$, with $v$ denoting kinematic fluid viscosity, and remains constant at $R e=300$ throughout this study, as in the work of Zhao and Cheng (2014). Although fluid flow at $R e=300$ is already in the turbulent regime, the relatively low $R e$ makes it affordable to directly solve the Navier-Stokes equations, which avoids the potential uncertainties associated with the utilisation of turbulence models. It is expected that the results obtained from the present work may help shed light on the effects of base column on the VIV response of real-world cylindrical structures under high $R e$ flow conditions. The VIV of the cylinder is simulated for a series of reduced velocities. The reduced velocity $V_{r}$ in this study is defined as $V_{r}=U /\left(f_{n} D\right)$, where $f_{n}$ is the natural frequency of the cylinder in vacuum. In order to keep $R e$ unchanged for different cases while $V_{r}$ is varied, only $f_{n}$ or equivalently, the stiffness $k$ of the spring restraining the cylinder, is adjusted.

\section{Numerical methods}

\subsection{Flow model}

The flow past a freely oscillating cylinder is assumed to be transient, incompressible and viscous, and is governed by the following Navier-Stokes equations formulated in an Arbitrary Lagrangian-Eulerian (ALE) framework:

$$
\begin{gathered}
\nabla \cdot \mathbf{U}=0 \\
\frac{\partial \mathbf{U}}{\partial t}+\nabla \cdot\left[\left(\mathbf{U}-\mathbf{U}_{g}\right) \mathbf{U}\right]=-\frac{\nabla p}{\rho}+\nu \nabla^{2} \mathbf{U}
\end{gathered}
$$


where $\mathbf{U}$ and $\mathbf{U}_{g}$ represent the velocity vector of flow field and computational grid, respectively; $t$ is time; $p$ is the pressure of flow field; $\rho$ is fluid density; $v$ denotes the kinematic viscosity of fluid.

The flow field is solved using OpenFOAM version 4.1 (https://openfoam.org), which is an open source CFD code and employs a finite volume method to discretise the governing equations. OpenFOAM has a large number of solvers with various functionalities available and the pimpleDyMFoam solver with a moving mesh capability is selected, where a PIMPLE (merged PISO-SIMPLE) algorithm is applied to deal with the coupling of velocity and pressure in a segregated way. A backward scheme with second-order accuracy is used for temporal discretisation while a secondorder upwind scheme is adopted for convective terms. Gradient terms are handled via a second-order Gauss linear scheme.

\subsection{Structural model}

The transverse response of an elastically mounted cylinder subjected to fluid forces can be described via the following equation of motion:

$$
m \ddot{y}+c \dot{y}+k y=F_{y}
$$

where $y$ denotes the transverse motion of the cylinder; $m, c$ and $k$ represent the cylinder mass, structural damping coefficient and spring stiffness, respectively; $F_{y}$ stands for the fluid force exerted upon the cylinder in the transverse direction.

In this study, structural damping is not considered and thus the damping coefficient $c$ is set to zero. The Newmark-beta method (Newmark, 1959) widely used for structural dynamics problems is adopted to solve Eq. (3) for the transverse response $y$ of the cylinder. The integration parameters are set to $\gamma=0.5$ and $\beta=0.25$ to ensure that the method is unconditionally stable and second-order accurate.

\subsection{Fluid-structure interaction}

The interaction between fluid and the cylinder is handled in a coupled manner as illustrated in Figure 3. When a simulation starts, the flow field surrounding the cylinder is firstly initialised. At the beginning of each time step, the fluid force acting on the cylinder is computed by integrating the pressure and shear stress over the cylinder 
surface. The response of the cylinder is then calculated by solving Eq. (3). Subsequently, the computational mesh is updated in a solid body motion manner, i.e. the whole mesh translates along with the cylinder so that grid quality remains unchanged. The NavierStokes equations, i.e. Eq. (1)-(2), are solved afterwards to update the flow field. The calculation procedure is then repeated for every time step until the end of the simulation.

\section{Validation tests}

The FSI tool presented in Section 3 is validated by studying the following two VIV cases and comparing the results from present simulations with published data.

\subsection{VIV of a 2D circular cylinder at $R e=150$}

The first validation case is the VIV of a circular cylinder at $R e=150$, which has been investigated previously in several numerical studies (Bao et al., 2011; Zhao, 2013; Wang et al., 2019). The mass ratio $m^{*}$ is set to 2.546 and the structural damping coefficient $c$ is zero. Only the cylinder response in the transverse direction is considered. As the Reynolds number is rather low, the flow is in the laminar regime and vortex shedding behind the cylinder is effectively two-dimensional (Kim and Choi, 2005). Thus, 2D simulations are carried out. A series of test cases is set up by varying the reduced velocity $V_{r}$ from 2 to 9 with an increment of 0.5 .

Figure 4 shows the non-dimensional transverse response amplitude $A_{y} / D$ of the cylinder as well as the mean and rms of the fluid force coefficients, including $C_{D, \text { mean }}$, $C_{D, r m s}$ and $C_{L, r m s}$, at various $V_{r}$. It is shown in Figure 4(a) that the transverse response amplitude $A_{y}$ reaches its maximum of around 0.6 times the cylinder diameter at $V_{r}=4$ with a sharp increase from less than $0.1 D$ at $V_{r}=3$, then stays above $0.4 D$ until $V_{r}=7.5$, followed by a significant decrease to $0.1 D$. Considerable variations of the force coefficients with respect to $V_{r}$ are also present from Figure 4(b)-(d) with their maxima occurring at about $V_{r}=4$, which is in accordance with $A_{y} / D$. A good agreement is achieved between the results calculated using the present tool and those obtained from the three existing studies, which demonstrates that the numerical methods established in this study are capable of accurately predicting the VIV of a circular cylinder at low Reynolds numbers of $R e<200$. 


\subsection{VIV of a circular cylinder with a finite length at $R e=300$}

In the second validation test, the VIV of a circular cylinder with a finite length at $R e=300$ is simulated, which was previously studied by Zhao and Cheng (2014) to analyse the effects of the free end of the cylinder on its response. No base column is attached and thus the diameter of the cylinder is constant. The aspect ratio $L / D$ of the truncated cylinder is set to 2 , with a low mass ratio $m^{*}$ of 2 and zero structural damping. The cylinder is only allowed to translate in the transverse direction. Three-dimensional simulations are performed for this case due to the intrinsic turbulent characteristics of flow at $R e=300$ and the three-dimensionality of the geometry. The reduced velocity $V_{r}$ is varied from 3 to 12 with an increment of 1.

Figure 5 compares $A_{y} / D$, response frequency ratio $f / f_{n}$ and $C_{L, r m s}$ at different $V_{r}$ from the present simulations with the predictions from Zhao and Cheng (2014). The largest transverse response amplitude $A_{y, \max }$ appears at $V_{r}=6$ with a value of about $0.75 D$, as shown in Figure 5(a). Then $A_{y}$ gradually decreases to $0.25 D$ at $V_{r}=12$. Figure 5(b) shows that the frequency $f$ of the cylinder response becomes close to its natural frequency in vacuum $f_{n}$ as $V_{r}$ increases while $C_{L, r m s}$ in Figure 5(c) peaks at $V_{r}=5$ and remains relatively small outside the range of $4 \leq V_{r} \leq 7$. The results from the two simulations agree rather well with each other, which further validates the present tool for VIV studies with turbulent and three-dimensional flow characteristics.

\section{Computational model}

The computational model established for the VIV simulation of the present circular cylinder with a base column is described in this section.

\subsection{Computational domain and boundary conditions}

Figure 6 illustrates the overall computational domain used in the present study. The top of the cylinder is located on the top side of the domain while the distance from the bottom of the base column to the domain bottom is $10 D$, in which $D$ denotes the diameter of the upper part of the cylinder. The centreline of the cylinder is $20 \mathrm{D}$ away from the other two sides as well as the Inlet boundary, and $30 D$ from the Outlet boundary, so as to minimise the influence of domain boundaries on the cylinder. 
The boundary conditions for all the boundaries of the domain are also annotated in Figure 6. Constant flow speed $U$ in the $x$ direction is specified for the fluid velocity $\mathbf{U}$ at the Inlet boundary while the fluid pressure $p$ at the Outlet boundary is set to zero. A symmetry boundary condition is applied at the four side boundaries, where the velocity component normal to the boundaries is zero. The effect of free surface on the cylinder is not considered in this study. A moving wall boundary condition is applied to the cylinder surface so that the fluid velocity $\mathbf{U}$ on the surface is equal to the predicted velocity of the structure $\mathbf{U}_{s}$.

\subsection{Mesh and time-step size sensitivity tests}

It is well known that the density of the computational mesh used for CFD simulations has large impacts on numerical results. Thus, a mesh sensitivity test is carried out by setting up three cases with different mesh density, named Coarse, Medium and Fine as listed in Table 1. All meshes adopt a same structured grid topology but with different number of elements assigned, leading to different overall cell count. Specifically, the number of elements along the circumference of the cylinder for the three meshes is set to 60,80 and 100, respectively. The height of the first cell away from the cylinder surface is $0.004 D$ for all the grids, which is found to be sufficiently small by Zhao and Cheng (2014) in their simulations of finite-length cylinders similar to the present study. Figure 7 shows the computational mesh for the Medium grid, focusing on the top side of the domain and the cylinder surface. It can be easily seen that grid points are distributed towards the surface of the structure to better capture vortex shedding as well as the interaction between fluid flow and the structure. A same flow condition with a reduced velocity $V_{r}$ of 6 is applied to all the three cases. This is the same $V_{r}$ at which the VIV response amplitude peaks for the cylinder with a finite length in Section 4.2. Considering the similarities between the two configurations, it is reasonable to expect significant VIV responses for the present study at $V_{r}=6$, which is ideal for a mesh sensitivity test. Figure 8 compares the time series of the nondimensional cylinder response $y / D$ and lift coefficient $C_{L}$ within two periodic response cycles for the three cases. It is shown that the curves from the Medium and Fine meshes nearly coincide with each other, indicating that the mesh density adopted for the Medium grid is adequate for the present study. The time series data is further 
processed into $A_{y} / D$ and $C_{L, r m s}$, as compared in Table 1 . In order to quantitatively assess differences among the results from the three meshes and numerical uncertainties associated with the present simulations, the relative error between two adjacent grid levels and Grid Convergence Index (GCI) (Roache, 1994) are calculated and listed in Table 2. The small value of GCI and its reduction for successive mesh refinements indicate that a numerical solution insensitive to cell size and with low numerical uncertainty is obtained. In order to achieve a balance between the accuracy of results and computational cost, the Medium grid is selected for subsequent simulations.

Similarly, a sensitivity test is conducted for the time-step size $\Delta t$ to determine its optimal value. A nondimensional time-step size $\Delta t / T_{v}$ is defined by dividing $\Delta t$ with the vortex shedding period $T_{v}$ for a stationary, infinitely long cylinder. $T_{v}$ is related to the Strouhal number $S t$ and can be calculated as $T_{v}=D /(S t \cdot U)$ while $S t$ is about 0.2 at $R e=300$ (Zhao and Cheng, 2014). Three simulations are performed with $\Delta t / T_{v}$ set to $1 / 500,1 / 1000$ and 1/2000, respectively, as summarised in Table 1. All other parameters including the computational mesh are kept the same. The comparison of the time series data in Figure 9 reveals very good agreement for $\Delta t / T_{v}=1 / 1000$ and 1/2000, which is further corroborated in Table 1 by the close values of $A_{y} / D$ and $C_{L, r m s}$ between the two cases. Similar to the above mesh-sensitivity test, relative error and GCI are also computed with the three levels of time-step size. The results listed in Table 2 show that the time-step size of $\Delta t=T_{v} / 1000$ is sufficiently small and is thus adopted in the present study.

\section{Results and discussion}

This section presents the results obtained for the cylinder with the base column under stationary and oscillating conditions. Detailed analysis is carried out by comparing the present results with those from the finite cylinder without the base column previously studied in Section 4.2.

\subsection{Flow past a stationary cylinder with a base column}

The flow past a stationary cylinder with a base column at $R e=300$ is simulated in this section to study the flow characteristics associated with the present geometry. Results of force coefficients and flow field are presented and analysed. 


\subsubsection{Force coefficients}

Figure 10 shows the time series of the force coefficients $C_{D}$ and $C_{L}$ for the cylinder with the base column. The force coefficients are obtained by nondimensionalising the in-line and transverse forces $F_{x}$ and $F_{y}$ in the following way:

$$
C_{D}, C_{L}=\frac{F_{x}, F_{y}}{0.5 \rho U^{2} A_{p}}
$$

where $\rho$ is fluid density; $U$ is flow speed; $A_{p}$ is the projected area of the structure on the plane normal to the flow direction and is defined as $A_{p}=D L_{C}+D_{B} L_{B}$ (refer to Figure 1). Time $t$ is also non-dimensionalised by the flow speed $U$ and the diameter of the upper cylinder $D$ as $U t / D$. It is easily seen that the variation of both coefficients with respect to time is rather small. Specifically, $C_{D}$ remains nearly constant at about 0.9 and $C_{L}$ stays close to zero throughout the simulation, which is in stark contrast with the periodically fluctuating results for an infinitely long cylinder at the same Reynolds number of 300 , where $C_{D, \text { mean }} \approx 1.32$ and $C_{L, r m s} \approx 0.46$ (Kim and Choi, 2005). This, however, is in accordance with the results of the finite cylinder without the base column as also shown in Figure 10, where the force coefficients are found to barely oscillate with $C_{D \text {,mean }} \approx 0.8$ and $C_{L, r m s} \approx 0$. The results suggest that the periodic vortex shedding typically present in the flow past an infinitely long cylinder does not occur for the cylinder with the base column, implying that the free end effects may still exist even with the presence of the base column.

\subsubsection{Fluid flow}

Figure 11(a)-(b) show the vortex flow in the wake of the stationary cylinder with the base column at $R e=300$ from two different views. The three-dimensional vortex structure is represented by the iso-surface of $Q=0.2$, where $Q$ is the second invariant of the rate of strain tensor. A pair of separated shear layers (termed "Shear layer" in Figure 11) are generated from the two sides of the upper cylinder and extend to the downstream of the structure. Additionally, a pair of vortex tubes (termed "Upper edge vortex") parallel to the incident flow are clearly visible, which originate from the two sides of the upper edge of the base column and merge with the shear layers in the downstream. Similar to the shear layers in the upper part, vortices (termed "Side vortex") 
also form from the sides of the base column but fail to develop further possibly due to the relatively short length of the base column. When viewed from the bottom, another vortex tube (termed "Lower edge vortex") generated from the lower edge of the base column can be easily discerned, which bends upward to the positive $z$ direction. Moreover, a large vortex (termed "Bottom vortex") covering the bottom of the base column is present, which is typically referred to as the arch or mushroom vortex (Sumner, 2013). The vortex patterns associated with a stationary finite cylinder without the base column are also illustrated in Figure 11(c)-(d). Comparing the vortices from the two simulations reveals some similarities, e.g. the presence of the edge vortex and its merging with the shear layer. Meanwhile, more complex vortex flow is also observed for the case with the base column. Apart from the additional "Upper edge vortex" and "Side vortex", it is believed that attaching a base column to the finite cylinder also leads to the merging of the pair of "Trailing edge vortices" displayed in Figure 11(d), which are generated from the two sides of the bottom edge similar to the "Upper edge vortex", into the single "Lower edge vortex" shown in Figure 11(b).

Another notable feature of the vortex flow in Figure 11(a)-(b) is that it is mostly symmetric about the midplane $y=0$ with no asymmetric patterns such as von Kármán vortex streets observed, which is in accordance with the findings of Benitz et al. (2016) and substantiates the barely noticeable variations in the force coefficients plotted in Figure 10. This is further corroborated by visualising the local vorticity field at different spanwise locations. Slice planes perpendicular to the axial axis of the cylinder are constructed as illustrated in Figure 12. A non-dimensional length $z / L$ is defined to determine the location of a slice plane by dividing the vertical coordinate $z$ with the overall length of the cylinder $L$, with $z / L=0$ being the bottom of the base column and $z / L=1$ the top of the cylinder. Figure 13 shows the contours of the axial vorticity $\omega_{z}$ for the stationary cylinder with the base column at $R e=300$ on four slice planes along the cylinder, i.e. $z / L=0.1,0.25,0.5$ and 0.95 . It can be seen from Figure 13 that symmetric shear layers separate from the two sides of the cylinder and stretch out to the downstream of the cylinder with no apparent vortex shedding observed within the distance of up to about $5 D$. It is worth pointing out that in Figure 13(c), where the slice plane $z / L=0.25$ is close to the base column, the pair of shear layers associated with 
the upper cylinder gradually spread outward and merge with the vortices generated from the upper edge of the base column as discussed earlier.

The suppression of periodic vortex shedding in the wake of the stationary cylinder indicates that attaching a base column with the current dimensions to the cylinder does not seem to prevent the free end effects from influencing the fluid flow around the structure. This is further demonstrated in Figure 14 by representing the flow with streamlines coloured by non-dimensional vertical velocity at the midplane $y=0$ section for the cylinder with and without the base column. A large recirculation zone, which is characteristic of fluid flow associated with a free end (Sumner, 2013), is observed behind the cylinder regardless of the presence of the base column, clearly illustrating the effects of free end on the flow around the cylinder with a base column.

\subsection{VIV of an elastically mounted cylinder with a base column}

The VIV of the elastically mounted cylinder with the base column at $\operatorname{Re}=300$ is analysed in this section from the following aspects: motion response, force coefficient and fluid flow. A series of simulations with different reduced velocity $V_{r}$, varying from 4 to 12 with an increment of 1 , is performed.

\subsubsection{Motion response}

Figure 15(a) shows the variation of the non-dimensional transverse response amplitude $A_{y} / D$ with respect to $V_{r}$. It is found that $A_{y} / D$ is nearly zero at $V_{r}=4$ and quickly rises to its maximum of around 0.6 at $V_{r}=6$ then gradually decreases to about 0.17 at $V_{r}=12$. The result from a finite-length cylinder without the base column is also plotted in Figure 15(a). Comparing the two curves reveals that although they are similar in shape and both peak at $V_{r}=6$, the largest response amplitude $A_{y, \max }=0.6 \mathrm{D}$ for the cylinder with the base column is noticeably smaller than $0.75 \mathrm{D}$ for the case without the base column, indicating the great impacts of attaching the base column on the cylinder response. Figure 15(b) compares the response frequency ratio $f / f_{n}$ between the two cases. For simulations with irregular responses, a spectral analysis is conducted for the response time series and the frequency with the greatest spectrum magnitude is considered the dominant response frequency $f$. A similar trend is observed in Figure 15(b) with both curves increasing almost linearly from $V_{r}=6$ to $V_{r}=11$ and crossing 
the line $f / f_{n}=1$. It is also found that $f / f_{n}$ for the cylinder with the base column is generally smaller than that for the case without the base column. Although the difference is less than 5\% as compared in Table 3, it still affects the response amplitude of the cylinder as will be shown later in Section 6.2.2.

Khalak and Williamson (1999) found that for the VIV of an elastically mounted cylinder with a low mass-damping $m^{*} \zeta$, there are three distinct response modes, i.e. an initial branch, an upper branch and a lower branch. As no structural damping is considered in the present study, i.e. $m^{*} \zeta=0$, these three branches are expected to be found for the cylinder with the base column as well. The transition between the branches was found to relate to a jump in the response frequency ratio (Govardhan and Williamson, 2000). Specifically, the transition between the initial and upper branches is marked by the jump of the response frequency ratio $f / f_{m}$ across 1.0 while the upperlower transition occurs when $f / f_{n}$ passes through 1.0, where $f_{m}$ and $f_{n}$ are the natural frequencies of the cylinder in fluid and vacuum, respectively. Apart from $f / f_{n}$, the response frequency ratio $f / f_{m}$ is also plotted in Figure 15(b). It is shown that the cylinder response transits from the initial branch to the upper branch between $V_{r}=5$ and $V_{r}=6$ for both cases. The transition between the upper and lower branches takes place near $V_{r}=9$, i.e. $V_{r}=9 \sim 10$ and $V_{r}=8 \sim 9$ for the cylinder with and without the base column, separately. Therefore, the $V_{r}$ range for the upper branch is determined as $6 \leq V_{r} \leq 9$ for the cylinder with the base column, which is slightly larger than $6 \leq V_{r} \leq 8$ for the case without the base column.

Another indicator for the transition between branches is the jump in the phase difference between the cylinder response and the fluid force. Two phase angles are involved: $\phi_{\text {vortex }}$, defined as the phase difference between the vortex force $F_{\text {vortex }}$ and the response $y$; and $\phi_{\text {total }}$, which is the phase difference between the total fluid force $F_{\text {total }}$ or $F_{y}$ and the response $y$. The vortex force $F_{\text {vortex }}=F_{\text {total }}-F_{\text {potential }}$ is computed by subtracting the potential added-mass force $F_{\text {potential }}$ from $F_{\text {total }}$ while $F_{\text {potential }}$ is calculated as $F_{\text {potential }}=-C_{A} m_{d} \ddot{y}$, in which $C_{A}$ is the added mass coefficient taken as 1 for a circular cylinder and $m_{d}$ is the displaced fluid mass. Instantaneous phases between the forces and the response are obtained via the Hilbert transform and averaged over time. Figure 16 plots the time-averaged $\phi_{\text {vortex }}$ and $\phi_{\text {total }}$ with respect to $V_{r}$ for the 
VIV of the cylinder with and without the base column. According to Govardhan and Williamson (2000), the $180^{\circ}$ jump of $\phi_{\text {vortex }}$ between $V_{r}=5$ and $V_{r}=6$ for both cases, as shown in Figure 16(a), denotes the transition between the initial and upper branches. Meanwhile, in Figure 16(b), $\phi_{\text {total }}$ jumps from $0^{\circ}$ to about $180^{\circ}$ at $V_{r}=9 \sim 10$ and $V_{r}=8 \sim 9$ for the cylinder with and without the base column, respectively, signifying the upper-lower transition. It is noted that the transition ranges are in accordance with the analysis of the response frequency ratios in Figure 15(b).

The lock-in or synchronisation regime is then determined, which is marked by a lower boundary (smallest $V_{r}$ ) and an upper one (largest $V_{r}$ ). The lower boundary can be easily defined from Figure 15(a), where a step change in $A_{y} / D$ occurs near the initial-upper response transition, i.e. $V_{r}=6$ for the case with the base column and $V_{r}=5$ for that without the base column. The upper boundary, however, is difficult to determine visually as the transition from the lower branch to the desynchronised regime is not evident in Figure 15(a). In order to locate the upper boundary of the lock-in regime, the definition of lock-in proposed by Williamson and Govardhan (2004) is adopted, i.e. the matching of the cylinder response frequency $f$ with that of periodic vortex shedding, or equivalently, the frequency of the fluid force exerted on the cylinder. Figure 17 presents the time series and power spectral density (PSD) of $y / D$ and $C_{L}$ for the VIV of the cylinder with the base column at three different $V_{r}$. The plots at $V_{r}=6$ show a typical example within the lock-in regime, with a significant response amplitude and a very good matching of response and force frequencies. Despite the substantial variations in the time series of $C_{L}$ at $V_{r}=11$, its dominant frequency is equal to that of the cylinder response. However, the PSD plot of $C_{L}$ at $V_{r}=12$ has multiple peaks and its dominant frequency deviates from that of the response, indicating that the response and force are desynchronised. As a result, $V_{r}=11$ is regarded the upper boundary of the lock-in regime for the cylinder with the base column, with the whole range determined as $6 \leq V_{r} \leq 11$. Similarly, in order to find the upper boundary of the lock-in regime for the cylinder without the base column, the time series and PSD of $y / D$ and $C_{L}$ at $V_{r}=9$ and $V_{r}=10$ are plotted in Figure 18. By comparing the response and force frequencies from the PSD plots, it is easily seen that $V_{r}=9$ indicates the upper boundary of the lock-in regime while $V_{r}=10$ is already in the 
desynchronised regime although $A_{y} / D$ at $V_{r}=10$ is still above 0.3 . Therefore, the lock-in regime for the cylinder without the base column is $5 \leq V_{r} \leq 9$. The $V_{r}$ range for the transition between branches to occur and the lock-in regime is summarised in Table 4 for the two cases. By comparing the lock-in regime between the two cases, it is found that the range of $V_{r}$ where lock-in occurs expands and is shifted towards the larger $V_{r}$ end when the base column is attached to the cylinder.

\subsubsection{Force coefficient}

Figure 19 compares the variation of $C_{L, r m s}$ with respect to $V_{r}$ between the cylinder with and without the base column. For the finite cylinder without the base column, $C_{L, r m s}$ increases rapidly and reaches its maximum at $V_{r}=5$, followed by a sudden drop. Govardhan and Williamson (2000) also found in their experiment of long cylinders with high $L / D$ that the maximum lift coefficient occurs at the initial-upper transition. This can be explained by the fact that the vortex force and potential added-mass force in the initial branch are in phase with each other, as supported by the nearly zero $\phi_{\text {vortex }}$ shown in Figure 16(a), resulting in a relatively large total force at $V_{r}=5$. Once the two force components are out of phase by $180^{\circ}$, they cancel each other out, leading to an overall small total force. Although Figure 19 show that $C_{L, r m s}$ peaks at $V_{r}=6$ for the cylinder with the base column, considering the similarity between the two cases in the variation of $\phi_{\text {vortex }}$ illustrated in Figure 16(a), it is thus reasonable to expect that an even larger $C_{L, r m s}$ would appear between $V_{r}=5$ and $V_{r}=6$, where the cylinder response transits from the initial to the upper branch. This is substantiated by reducing the increment for $V_{r}$ and achieving a larger $C_{L, r m s}$ of 0.64 at $V_{r}=5.4$ in the initial branch.

Further examining $A_{y} / D$ in Figure 15(a) and $C_{L, r m s}$ in Figure 19 for the two cases at $V_{r}=6$ raises a question: why does $A_{y} / D$ become smaller while $C_{L, r m s}$ is larger, when the base column is attached to the cylinder? In order to establish a relationship between $A_{y} / D$ and $C_{L, r m s}$, a sinusoidal form is assumed for the transverse response $y$ and force $F_{y}$ in the lock-in regime:

$$
\begin{gathered}
y=A_{y} \sin (\omega t) \\
F_{y}=F_{0} \sin \left(\omega t+\phi_{\text {total }}\right)
\end{gathered}
$$


where $\omega=2 \pi f$ is the angular frequency of the cylinder response and $F_{0}$ is the amplitude of $F_{y}$. The frequency of $F_{y}$ is equal to that of $y$ according to the definition of lock-in mentioned earlier. Substituting Eq. (5)-(6) into Eq. (3) leads to:

$$
\left(k-m \omega^{2}\right) A_{y} \sin (\omega t)=F_{0} \sin \left(\omega t+\phi_{\text {total }}\right)
$$

It should be noted that as structural damping is not considered in this study, the associated term is removed from Eq. (7). It immediately becomes apparent that $\phi_{\text {total }}$ should be equal to $0^{\circ}$ or $180^{\circ}$ with no structural damping present, which is evidenced by Figure 16(b). Reorganising Eq. (7) gives the following relation between $A_{y}$ and $F_{0}$ :

$$
A_{y}=\frac{F_{0}}{m\left|\omega_{n}^{2}-\omega^{2}\right|}
$$

where $\omega_{n}=2 \pi f_{n}=\sqrt{k / m}$ is the natural angular frequency of the cylinder in vacuum and the absolute value sign is associated with the jump in $\phi_{\text {total }}$ from $0^{\circ}$ to $180^{\circ}$. By substituting a series of non-dimensional parameters into Eq. (8), i.e. $V_{r}, m^{*}$ and $C_{L 0}$ (non-dimensionalised $F_{0}$ via Eq. (4)), and employing the relation $C_{L, r m s}=C_{L 0} / \sqrt{2}$ which is valid for a sinusoidal signal, $A_{y} / D$ is found to relate to $C_{L, r m s}$ for the cylinder with the base column in the following form:

$$
A_{y} / D=\frac{\sqrt{2}}{2 \pi^{3}} \frac{C_{L, r m s} V_{r}^{2}}{m^{*}\left|1-\left(f / f_{n}\right)^{2}\right|} \frac{1+\left(D_{B} / D\right)\left(H_{B} / H_{C}\right)}{1+\left(D_{B} / D\right)^{2}\left(H_{B} / H_{C}\right)}
$$

It is evident from Eq. (9) that in addition to $V_{r}, m^{*}$ and $f / f_{n}$, the geometrical parameters of the structure, i.e. $D_{B} / D$ and $H_{B} / H_{C}$ as annotated in Figure 1, also play an important part in determining the relationship between $A_{y} / D$ and $C_{L, r m s}$. For a cylinder with a constant diameter, e.g. the one without the base column, the third fraction on the right side of Eq. (9) is equal to 1, while it is 0.75 for the cylinder with the base column analysed in the present study. In order to make sure that Eq. (9) can be used to describe the relationship between $A_{y} / D$ and $C_{L, r m s}$ obtained from the present simulations, the corresponding simulation parameters, including $V_{r}, m^{*}, f / f_{n}$ and $C_{L, r m s}$, are substituted into the equation, and then the estimated $A_{y} / D$ via Eq. (9) is compared with the predicted value from the numerical simulation. Table 5 lists the comparison for the two cases with and without the base column at $V_{r}=6$. Good agreement between the estimated and predicted values is achieved, considering that an 
ideal sinusoidal assumption is made in Eq. (5)-(6). It is therefore demonstrated that Eq. (9), more specifically the difference in the geometry of the two cylinders, may be used as an explanation to the question raised earlier. A closer look into Eq. (9) also reveals that as $f / f_{n}$ approaches 1 , the part $1 /\left|1-\left(f / f_{n}\right)^{2}\right|$ increases significantly towards infinity and thus even a small $C_{L, r m s}$ could excite a large $A_{y} / D$, which justifies the relatively large response amplitude in the lower branch as shown in Figure 15(a) despite the small $C_{L, r m s}$ displayed in Figure 19.

Nevertheless, another question remains: why does the response amplitude of the cylinder decrease in the lock-in regime when the base column is attached? By further comparing the geometry of the two structures, it is noted that attaching the base column introduces two additional surfaces with considerable area, i.e. the top and bottom sides of the base column. It is thus speculated that these two sides may produce substantial damping force and adversely affect the cylinder response. In order to assess the impacts of the base column, particularly its two sides, on the VIV response of the cylinder, the energy transfer between fluid and the cylinder is analysed, following Jauvtis and Williamson (2004) in their experimental study of the VIV of cylinders with high $L / D$.

Firstly, the surface grid of the structure is decomposed and grouped into three individual parts: sides (the top and bottom sides of the base column), base column (the remaining part of the base column without the two sides) and upper cylinder (the main component of the cylinder without the base column and its sides), and the fluid forces exerted upon these parts are calculated during the simulations. Figure 20(b) shows the time series of $C_{L}$ computed via Eq. (4) over a complete response cycle for the three parts, taking $V_{r}=6$ for example. Time $t$ is non-dimensionalised by the response period $T$. It should be pointed out that as the two sides are perpendicular to the axial direction of the cylinder and parallel to the incident flow, $C_{L}$ of the sides only comes from shear stress integration, while both pressure and shear stress contribute for the other two parts. It is seen from Figure 20(b) that $C_{L}$ of the sides and of the base column are mostly in phase with each other but are out of phase with that of the upper cylinder, indicating a change in the fluid flow close to the base column. 
Subsequently, a normalised rate of energy transfer $\dot{E}^{*}$ is computed for the three parts and displayed in Figure 20(c). $\dot{E}^{*}$ at any instant is defined as:

$$
\dot{E}^{*}=C_{L} \dot{y}^{*}
$$

where $\dot{y}^{*}=\dot{y} T / D$ is the non-dimensional velocity as depicted in Figure 20(a). The frequency of $\dot{E}^{*}$ is found to be twice that of the cylinder response, with each of the two cycles corresponding to the movement in either positive or negative transverse direction. The sign of $\dot{E}^{*}$ indicates how energy is transferred between fluid and the cylinder: a positive $\dot{E}^{*}$ denotes an energy transfer from fluid to the structure while a negative value means the opposite. A notable feature observed from Figure 20(c) is that although the sides and the base column occasionally input energy from fluid to the cylinder, they mostly contribute negatively to the response.

In order to quantify the contribution of the sides, the normalised energy transfer $E^{*}$ over a complete response cycle is computed in the following manner:

$$
E^{*}=\frac{1}{T} \int_{0}^{T} \dot{E}^{*} d t=\frac{1}{T} \int_{0}^{T} C_{L} \dot{y}^{*} d t
$$

It is worth mentioning that for the present mass-cylinder system without structural damping, the overall energy transfer from fluid to the cylinder over a cycle must be equal to zero so as to sustain a periodic response. Therefore, the positive energy transfer associated with the upper cylinder has to be completely offset by the energy dissipated from the sides and the base column. Results of $E^{*}$ for the three parts show that the sides account for around $40 \%$ of negative energy transfer, clearly demonstrating that the additional upper and lower sides of the base column have considerable damping effects on the cylinder response, which likely results in the decrease in its response amplitude.

The fluid force exerted upon the cylinder with the base column is further analysed by examining the sectional lift coefficient $C_{L}(z / L)$ on slice planes at different length $z / L$, which has been defined previously in Figure 12. $C_{L}(z / L)$ is calculated as:

$$
C_{L}(z / L)=\frac{F_{y}(z / L)}{0.5 \rho U^{2} D(z / L)}
$$

where $F_{y}(z / L)$ and $D(z / L)$ are the sectional transverse force and cylinder diameter at length $z / L$. It should be noted that there is a jump in $D(z / L)$ when $z / L$ passes 
through 0.2. $V_{r}=6$ is selected as an example and the time series of $C_{L}(z / L)$ on a number of slice planes are plotted in Figure 21, together with the cylinder response $y / D$. Two slice planes very close to each other, i.e. $z / L=0.195$ and $z / L=0.205$, are intentionally constructed to investigate the effects of attaching the base column to the cylinder at $z / L=0.2$ on $C_{L}(z / L)$. It is seen from Figure 21 that $C_{L}(z / L)$ at various $z / L$ is mostly periodic with the same oscillating frequency as $y / D$ but differs from each other in both amplitude and phase. A detailed analysis is carried out by calculating the total phase $\phi_{\text {total }}$ and the sectional $C_{L, r m s}$ at different $z / L$, as plotted in Figure 22. Results from the cylinder without the base column are also displayed in Figure 22 for comparison. Figure 22(a) shows that $\phi_{\text {total }}$ gradually increases from about $-50^{\circ}$ at the bottom of the cylinder to around $30^{\circ}$ at its top for the case without the base column. When the base column is attached, a sharp $60^{\circ}$ jump in $\phi_{\text {total }}$ is observed near the attachment location $z / L=0.2$, with $\phi_{\text {total }}=-45^{\circ}$ on the base column side and $\phi_{\text {total }}=15^{\circ}$ on the upper cylinder side. Afterwards, $\phi_{\text {total }}$ stays at about $15^{\circ}$ from $z / L=0.2$ to $z / L=0.5$, then slowly rises to approximately $40^{\circ}$ at $z / L=1$. It is interesting to note that the sectional lift coefficients at the two ends are out of phase by about $90^{\circ}$. Meanwhile, Figure 22(b) reveals a nearly constant $C_{L, r m s}=0.4$ along the cylinder without the base column. For the case with the base column, however, a sudden drop in $C_{L, r m s}$ is firstly observed at $z / L=0.1$, followed by a steep rise to its maximum to $C_{L, r m s}=0.8$ near the upper edge of the base column before falling sharply again to $C_{L, r m s}=0.6$ just above $z / L=0.2$. Subsequently, $C_{L, r m s}$ decreases to about 0.4 at $z / L=0.7$ and almost stays unchanged until the top end of the cylinder. Jauvtis and Williamson (2004) argued that the energy transfer over a response cycle is determined by $C_{L, r m s} \sin \phi_{\text {total }}$. Figure 22 thus shows that the normalised sectional energy transfer $E^{*}(z / L)$ is always negative at $0<z / L<0.2$ and remains positive at $z / L>0.2$ for the cylinder with the base column, which agrees well with the results presented in Figure 20(c). Conversely, for the case without the base column, $E^{*}(z / L)$ gradually switches its sign along its vertical direction. Additionally, the significant variations in $\phi_{\text {total }}$ and $C_{L, r m s}$ near $z / L=0.2$ clearly suggest that the presence of the base column greatly affects the flow past the cylinder, which will be discussed in the following section. 


\subsubsection{Fluid flow}

Figure 23 visualises the three-dimensional vortex evolution within one response cycle for the cylinder with the base column at $V_{r}=6$. Seven consecutive instants denoted as 'A' - 'F' are marked in Figure 23. The iso-surface of $Q=0.2$ is constructed to represent the complex vortical structures. Note that the cylinder is viewed from its bottom and the side of the cylinder in the positive $y$ direction is shown. Similar to the vortices depicted in Figure 11(a)-(b) for the stationary cylinder, the shear layer separated from the side of the upper cylinder merges with the vortex generated by the upper edge of the base column (instant 'A'). Just after the cylinder moves past its maximum in the positive transverse direction, vortex shedding starts for the upper cylinder while the upper edge vortex still adheres to the base column (instant 'B'). The incident flow then convects the spanwise vortex shed from the upper cylinder to the downstream, effectively elongating the vortex in the streamwise direction (instant ' $\mathrm{C}$ '). The upper edge vortex is finally shed (instant ' $\mathrm{D}$ ') but is quickly attracted to the lee side of cylinder (instant ' $E$ '). While the spanwise vortex is convected further downstream, the upper edge vortex keeps drawing energy from the near wake of the cylinder and grows into a dominant spanwise vortex (instants ' $F$ ' and ' $G$ '). The evolution of the vortices in the wake of the cylinder with the base column is similar to what Zhao and Cheng (2014) presented for the finite cylinder without the base column, indicating again that the free end effects still exist for the present study. This is further evidenced by the streamline plot shown in Figure 24, from which it is clearly seen that a large number of streamlines are bent towards the axial direction right after they move past the lower side of the base column. The twisted shape of these streamlines also illustrates the complex flow characteristics in the wake of the cylinder.

The fluid flow around the cylinder with the base column at $V_{r}=6$ is further investigated by visualising the vorticity field at different spanwise sections. Figure 25 illustrates the contours of the axial vorticity $\omega_{z}$ on four slice planes, i.e. $z / L=0.1,0.21$, 0.5 and 0.9 , at a series of instants defined in Figure 23. Near the top of the cylinder at $z / L=0.9$, a $2 \mathrm{P}$ vortex shedding mode is observed, which is characterised by the shedding of a pair of counter-rotating vortices from the cylinder within each half cycle. The second vortex is found to be much weaker compared to the first one. The $2 \mathrm{P}$ mode 
is usually associated with the upper branch of the VIV response, as is the case for $V_{r}=6$ in the present study. It is also seen that the first vortex splits into a number of smaller vortices as it travels downstream. At $z / L=0.5$, vortex shedding appears in a $2 \mathrm{C}$ mode, where two co-rotating vortices form in each half cycle as annotated for instants ' $\mathrm{B}$ ' and ' $\mathrm{F}$ ' in Figure 25. The 2C mode was firstly reported by Flemming and Williamson (2005) in the VIV of pivoted cylinders and is not spotted for elastically mounted cylinders with large aspect ratios. Meanwhile, although not presented in this work, the $2 \mathrm{C}$ mode is also found in the VIV simulation results of the finite cylinder without the base column. Therefore, it is likely that the $2 \mathrm{C}$ mode is associated with the free end effects. Additionally, the vortex shedding near the base column at $z / L=0.1$ also exhibits the 2P mode and the two counter-rotating vortices are comparable in strength, which is noticeably different from that at $z / L=0.9$. It is also worth pointing out that the timing of the vortex shedding close to the base column lags behind that near the top end of the cylinder, which is in accordance with the variation of sectional $\phi_{\text {total }}$ shown in Figure 22(a). As for the slice plane $z / L=0.21$, the vortices induced by the base column can be clearly observed due to their close proximity, which surround the inner circular cross-section. Moreover, the vorticity strength close to the cylinder is relatively weaker than that in the outer region, and the shear layer separated from the cylinder merges with the outer vortices before vortex shedding occurs. The presence of the base column is found to greatly affect the fluid flow around the upper cylinder, which possibly results in the sudden jump in sectional $\phi_{\text {total }}$ and $C_{L, r m s}$ near $z / L=0.2$ depicted in Figure 22.

\section{Conclusions}

In this paper, the VIV of an elastically mounted rigid cylinder with a base column in uniform flow at $R e=300$ is investigated numerically. The cylinder is relatively short with a low aspect ratio of $L / D=2$. The base column has a diameter of $D_{B}=2 D$ and a length of $L_{B}=0.2 L$. A low mass ratio of $m^{*}=2$ is adopted and no structural damping is considered. The effects of the base column on the cylinder are analysed, and conclusions are presented as follows.

Contrary to our initial speculation, the free end effects associated with a finite cylinder still exist, and the base column with the present dimensions does not seem to 
prevent fluid from flowing around the bottom of the cylinder. Flow visualisation for a stationary cylinder shows that periodic vortex shedding is suppressed. When the cylinder undergoes VIV, it is found that fluid is directed towards its axial direction after moving around the base column and streamwise vortices dominate the wake flow. For $V_{r}=6$ in the upper branch, vortex shedding generally exhibits a $2 \mathrm{P}$ mode while a $2 \mathrm{C}$ mode with two co-rotating vortices formed in each half cycle is observed at $z / L=0.5$.

Despite the presence of the free end effects, attaching the base column to the cylinder results in an expanded lock-in regime, which is also shifted towards the larger end of the $V_{r}$ range. For the VIV of the cylinder with the base column in the lock-in regime, it is found that $A_{y} / D$ is smaller than that of the normal finite cylinder although $C_{L, r m s}$ is larger. This is explained by establishing a relationship between $A_{y} / D$ and $C_{L, r m s}$, which takes into account the geometrical properties of the base column. Variations are also observed for the sectional lift coefficient at different $z / L$, with a sharp jump in amplitude and phase near $z / L=0.2$ where the base column is attached.

Another finding from the present study is that the base column exerts significant damping effects on the VIV response of the cylinder. By analysing the rate of energy transfer from fluid to structure among different parts of the cylinder, it is found that the base column mostly contributes negatively to the cylinder response, with the upper and lower sides of the base column accounting for about $40 \%$ of negative energy transfer. Attaching the base column introduces two additional sides with large surface area and produces extra viscous damping. It is therefore reasonable to expect that increasing the diameter of the base column will lead to a further reduction in $A_{y} / D$ of the cylinder, which may serve as a way to alleviate the potential VIM problem in FOWT designs.

\section{Acknowledgements}

This work was supported by the National Natural Science Foundation of China (Grant Nos. 51909255 and U1806229). The authors would also like to acknowledge the Center for High Performance Computing and System Simulation, Pilot National Laboratory for Marine Science and Technology (Qingdao), for kindly providing access to its HPC facility. 


\section{References}

Azadeh-Ranjbar V, Elvin N, Andreopoulos Y (2018). Vortex-induced vibration of finite-length circular cylinders with spanwise free-ends: Broadening the lock-in envelope. Physics of Fluids, 30(10), 105104.

Bao Y, Zhou D, Tu J (2011). Flow interference between a stationary cylinder and an elastically mounted cylinder arranged in proximity. Journal of Fluids and Structures, 27(8), 1425-1446.

Bearman PW (1984). Vortex Shedding from Oscillating Bluff Bodies. Annual Review of Fluid Mechanics, 16(1), 195-222.

Benitz MA, Carlson DW, Seyed-Aghazadeh B, Modarres-Sadeghi Y, Lackner MA, Schmidt DP (2016). CFD simulations and experimental measurements of flow past free-surface piercing, finite length cylinders with varying aspect ratios. Computers \& Fluids, 136, 247-259.

Bourdier S, Chaplin JR (2012). Vortex-induced vibrations of a rigid cylinder on elastic supports with end-stops, Part 1: Experimental results. Journal of Fluids and Structures, 29, 62-78.

Flemming F, Williamson CHK (2005). Vortex-induced vibrations of a pivoted cylinder. Journal of Fluid Mechanics, 522, 215-252.

Fukuoka H, Hirabayashi S, Suzuki H (2016). The effects of free surface and end cell on flow around a finite circular cylinder with low aspect ratio. Journal of Marine Science and Technology, 21(1), 145-153.

Gonçalves RT, Franzini GR, Rosetti GF, Meneghini JR, Fujarra ALC (2015). Flow around circular cylinders with very low aspect ratio. Journal of Fluids and Structures, 54, 122-141.

Gonçalves RT, Meneghini JR, Fujarra ALC (2018). Vortex-induced vibration of floating circular cylinders with very low aspect ratio. Ocean Engineering, 154, 234-251.

Govardhan R, Williamson CHK (2000). Modes of vortex formation and frequency response of a freely vibrating cylinder. Journal of Fluid Mechanics, 420, 85-130.

Hover FS, Davis JT, Triantafyllou MS (2004). Three-dimensionality of mode transition in vortex-induced vibrations of a circular cylinder. European Journal of Mechanics - B/Fluids, 23(1), 29-40.

Jauvtis N, Williamson CHK (2004). The effect of two degrees of freedom on vortexinduced vibration at low mass and damping. Journal of Fluid Mechanics, 509, 2362.

Khalak A, Williamson CHK (1999). Motions, forces and mode transitions in vortexinduced vibrations at low mass damping. Journal of Fluids and Structures, 13(7), 813-851.

Kim J, Choi H (2005). Distributed forcing of flow over a circular cylinder. Physics of Fluids, 17(3), 033103.

Kinaci OK, Lakka S, Sun H, Bernitsas MM (2016). Effect of tip-flow on vortex induced vibration of circular cylinders for $\operatorname{Re}<1.2^{*} 10^{5}$. Ocean Engineering, 117, 130-142. 
Kitagawa T, Fujino Y, Kimura K (1999). EFFECTS OF FREE-END CONDITION ON END-CELL-INDUCED VIBRATION. Journal of Fluids and Structures, 13(4), 499-518.

Morse TL, Govardhan RN, Williamson CHK (2008). The effect of end conditions on the vortex-induced vibration of cylinders. Journal of Fluids and Structures, 24(8), 1227-1239.

Navrose, Mittal S (2019). Intermittency in free vibration of a cylinder beyond the laminar regime. Journal of Fluid Mechanics, 870, R2.

Newmark NM (1959). A method of computation for structural dynamics. Journal of the engineering mechanics division, 85(3), 67-94.

Palau-Salvador G, Stoesser T, Fröhlich J, Kappler M, Rodi W (2009). Large Eddy Simulations and Experiments of Flow Around Finite-Height Cylinders. Flow, Turbulence and Combustion, 84(2), 239.

Roache PJ (1994). Perspective: A Method for Uniform Reporting of Grid Refinement Studies. Journal of Fluids Engineering, 116(3), 405-413.

Robertson A, Jonkman J, Masciola M, Song H, Goupee A, Coulling A, Luan C. Definition of the semisubmersible floating system for phase II of OC4. 2014.

Sarpkaya T (2004). A critical review of the intrinsic nature of vortex-induced vibrations. Journal of Fluids and Structures, 19(4), 389-447.

Sumner D (2013). Flow above the free end of a surface-mounted finite-height circular cylinder: A review. Journal of Fluids and Structures, 43, 41-63.

Wang E, Xiao Q, Incecik A (2017). Three-dimensional numerical simulation of twodegree-of-freedom VIV of a circular cylinder with varying natural frequency ratios at $\mathrm{Re}=500$. Journal of Fluids and Structures, 73, 162-182.

Wang E, Xu W, Gao X, Liu L, Xiao Q, Ramesh K (2019). The effect of cubic stiffness nonlinearity on the vortex-induced vibration of a circular cylinder at low Reynolds numbers. Ocean Engineering, 173, 12-27.

Williamson CHK, Govardhan R (2004). VORTEX-INDUCED VIBRATIONS. Annual Review of Fluid Mechanics, 36(1), 413-455.

Williamson CHK, Govardhan R (2008). A brief review of recent results in vortexinduced vibrations. Journal of Wind Engineering and Industrial Aerodynamics, 96(6), 713-735.

Zhao M (2013). Flow induced vibration of two rigidly coupled circular cylinders in tandem and side-by-side arrangements at a low Reynolds number of 150. Physics of Fluids, 25(12), 123601.

Zhao M, Cheng L (2014). Vortex-induced vibration of a circular cylinder of finite length. Physics of Fluids, 26(1), 015111-1-26.

Zhao M, Cheng L, An H, Lu L (2014). Three-dimensional numerical simulation of vortex-induced vibration of an elastically mounted rigid circular cylinder in steady current. Journal of Fluids and Structures, 50, 292-311.

Zhao W, Zou L, Wan D, Hu Z (2018). Numerical investigation of vortex-induced motions of a paired-column semi-submersible in currents. Ocean Engineering, 164, $272-283$ 
Table 1 Results of mesh and time-step size sensitivity tests for VIV simulations at $V_{r}=6: N_{c}$ - number of elements along cylinder circumference; $\Delta t$ - time-step size;

$T_{v}$-vortex shedding period for a stationary, infinitely long cylinder

\begin{tabular}{|l|l|c|l|l|l|}
\hline \multicolumn{1}{c|}{ Mesh } & Cell count & $N_{c}$ & $\Delta t / T_{v}$ & $A_{y} / D$ & $C_{L, r m s}$ \\
\hline Coarse & 324,288 & 64 & $1 / 1000$ & 0.597 & 0.547 \\
\hline \multirow{3}{*}{ Medium } & \multirow{2}{*}{631,000} & \multirow{2}{*}{80} & $1 / 500$ & 0.595 & 0.544 \\
\cline { 4 - 6 } & & & $1 / 1000$ & 0.588 & 0.539 \\
\cline { 4 - 6 } & & & $1 / 2000$ & 0.584 & 0.536 \\
\hline Fine & $1,119,780$ & 100 & $1 / 1000$ & 0.584 & 0.538 \\
\hline
\end{tabular}

Table 2 Relative error $|\varepsilon|$ and GCI for three levels of mesh and time-step size: subscripts 1,2, 3 represent different cases with 1 denoting finest mesh or smallest time-step size and 3 being coarsest mesh or largest time-step size

\begin{tabular}{|c|c|c|c|c|c|}
\hline & Parameter & $\left|\varepsilon_{23}\right|(\%)$ & $\left|\varepsilon_{12}\right|(\%)$ & $G I_{23}(\%)$ & $G C I_{12}(\%)$ \\
\hline \multirow{2}{*}{ Mesh } & $A_{y} / D$ & 1.53 & 0.68 & 3.40 & 1.52 \\
\cline { 2 - 6 } & $C_{L, r m s}$ & 1.48 & 0.19 & 3.30 & 0.41 \\
\hline \multirow{2}{*}{$\begin{array}{c}\text { Time-step } \\
\text { size }\end{array}$} & $A_{y} / D$ & 1.19 & 0.68 & 1.98 & 1.14 \\
\cline { 2 - 6 } & $C_{L, \text { rms }}$ & 0.93 & 0.56 & 1.55 & 0.93 \\
\hline
\end{tabular}

Table 3 Relative difference of $f / f_{n}$ between a cylinder with and without a base column

\begin{tabular}{|c|c|c|c|c|c|c|c|}
\hline$V_{r}$ & 5 & 6 & 7 & 8 & 9 & 10 & 11 \\
\hline Without base column & 0.75 & 0.88 & 0.93 & 0.96 & 1.01 & 1.04 & 1.06 \\
\hline With base column & 0.76 & 0.85 & 0.89 & 0.93 & 0.97 & 1.02 & 1.04 \\
\hline Relative difference $(\%)$ & 1.33 & -3.41 & -4.3 & -3.12 & -3.96 & -1.92 & -1.89 \\
\hline
\end{tabular}

Table 4 Comparison of $V_{r}$ range for transition between branches and lock-in regime for VIV of a cylinder with and without a base column

\begin{tabular}{|l|c|c|}
\hline & With base column & Without base column \\
\hline Initial-upper transition & $5 \leq V_{r} \leq 6$ & $5 \leq V_{r} \leq 6$ \\
\hline Upper-lower transition & $9 \leq V_{r} \leq 10$ & $8 \leq V_{r} \leq 9$ \\
\hline Lock-in regime & $6 \leq V_{r} \leq 11$ & $5 \leq V_{r} \leq 9$ \\
\hline
\end{tabular}

Table 5 Comparison of $A_{y} / D$ between predicted from numerical simulations and estimated via Eq. (9) for VIV of a cylinder with and without a base column at $V_{r}=6$

\begin{tabular}{|l|c|c|c|c|c|c|}
\hline & $V_{r}$ & $m^{*}$ & $f / f_{n}$ & $C_{L, r m s}$ & $\left(A_{y} / D\right)_{\text {predicted }}$ & $\left(A_{y} / D\right)_{\text {estimated }}$ \\
\hline With base column & 6 & 2 & 0.847 & 0.542 & 0.588 & 0.590 \\
\hline Without base column & 6 & 2 & 0.878 & 0.406 & 0.742 & 0.727 \\
\hline
\end{tabular}




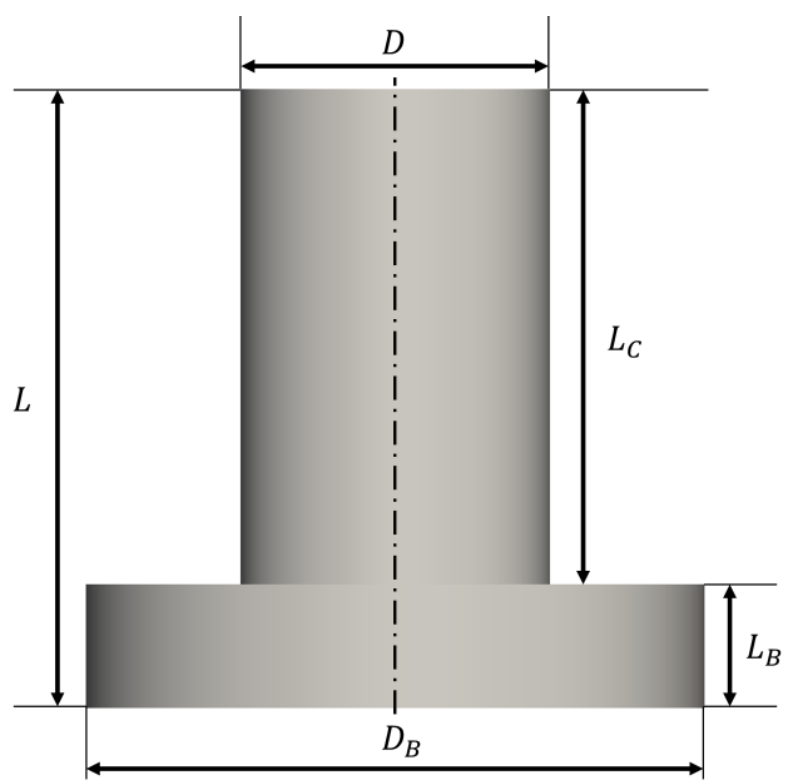

Figure 1 Definition of a circular cylinder with a base column: $L$ - overall length of cylinder; $D$ - diameter of upper cylinder; $L_{C}$ - length of upper cylinder; $D_{B}-$ diameter of base column; $L_{B}$ - length of base column

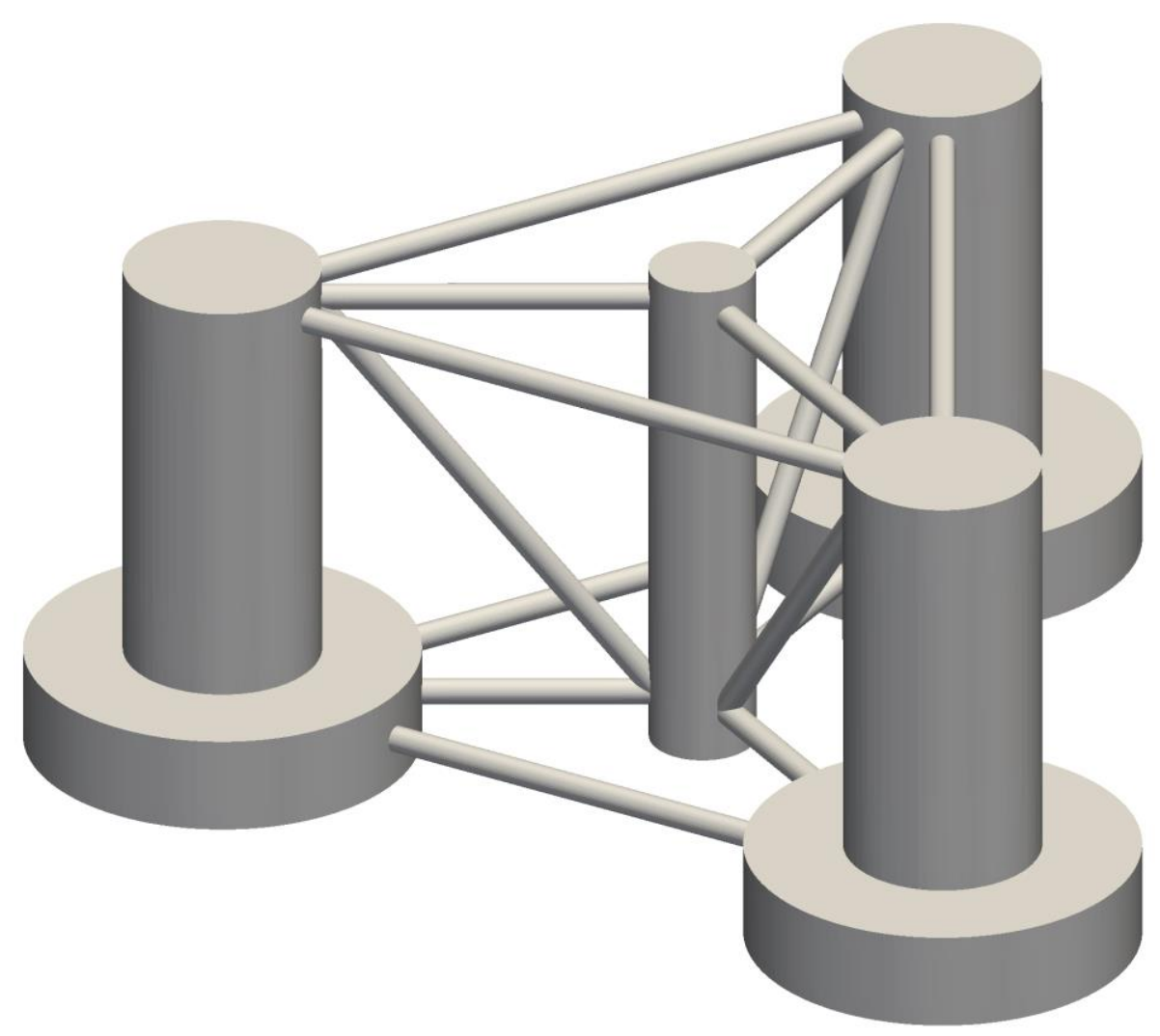

Figure 2 Illustration of DeepCwind semi-submersible platform for OC4 FOWT 


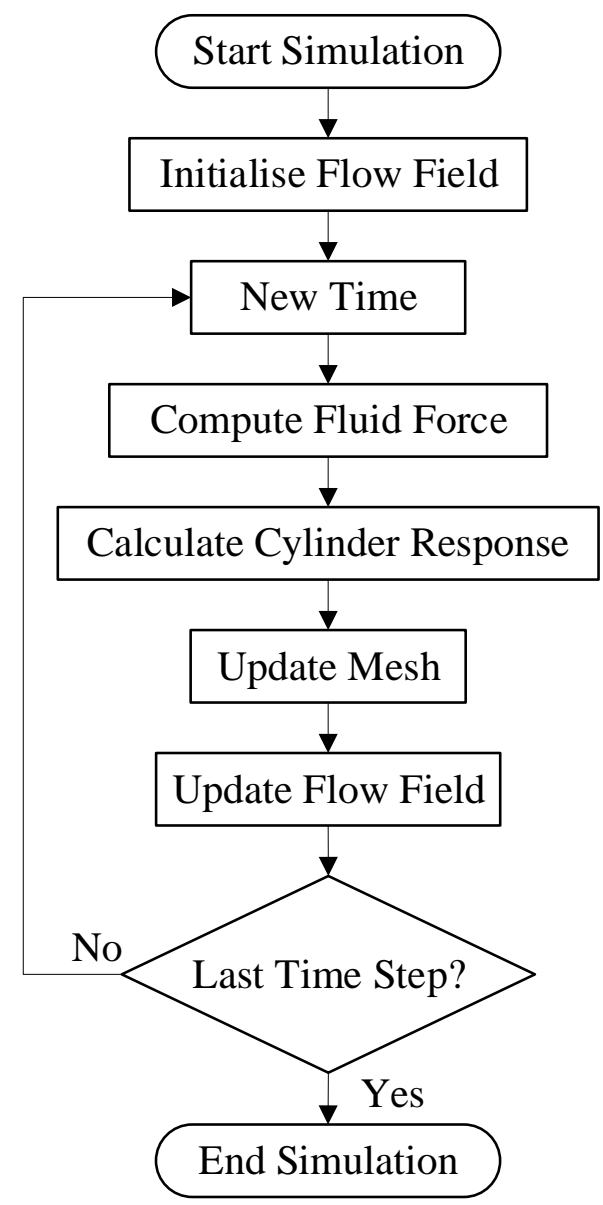

Figure 3 Flow chart of fluid-structure interaction 

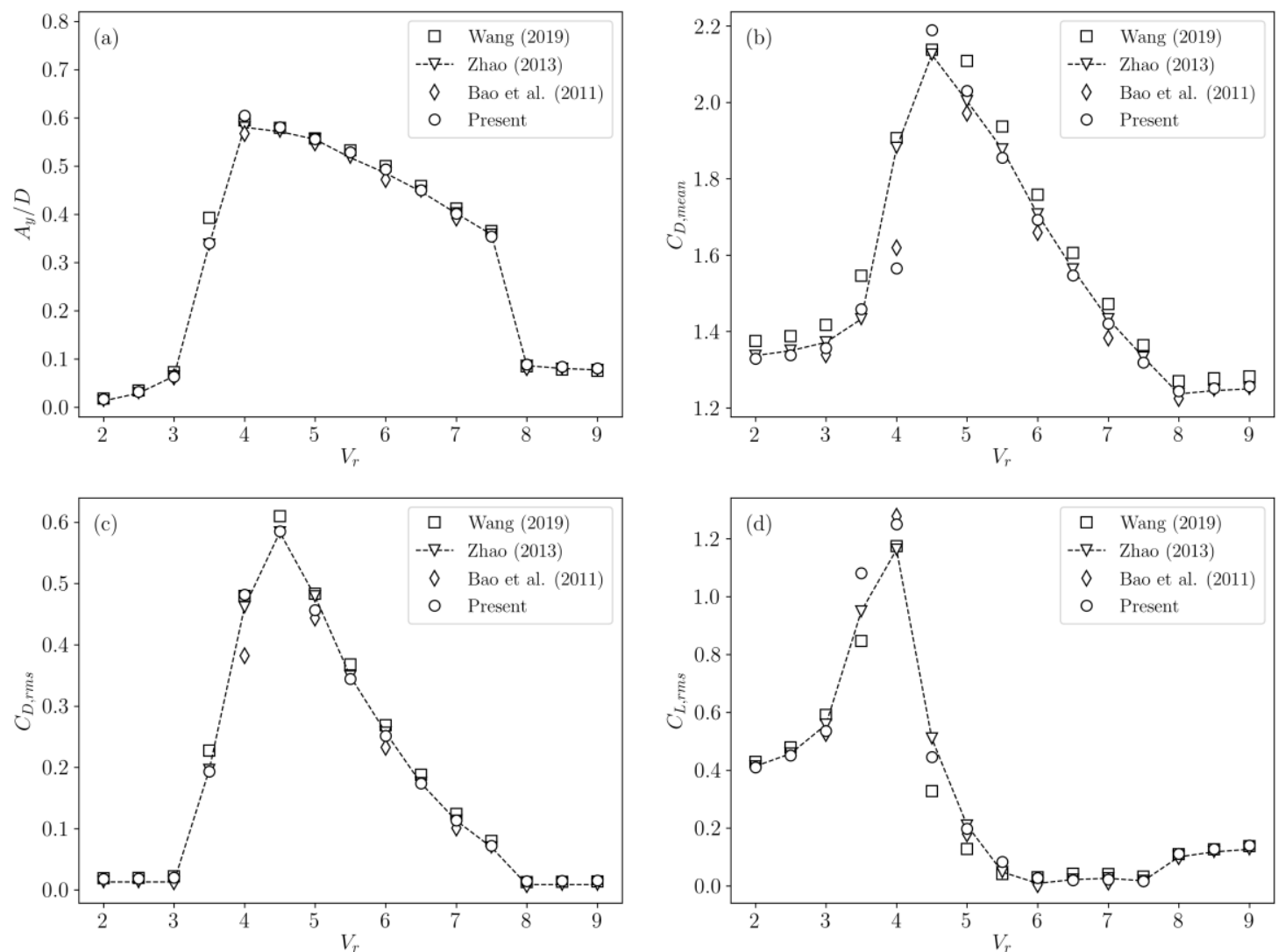

Figure 4 Response amplitude and force coefficients of VIV of a 2D circular cylinder at $\operatorname{Re}=150$
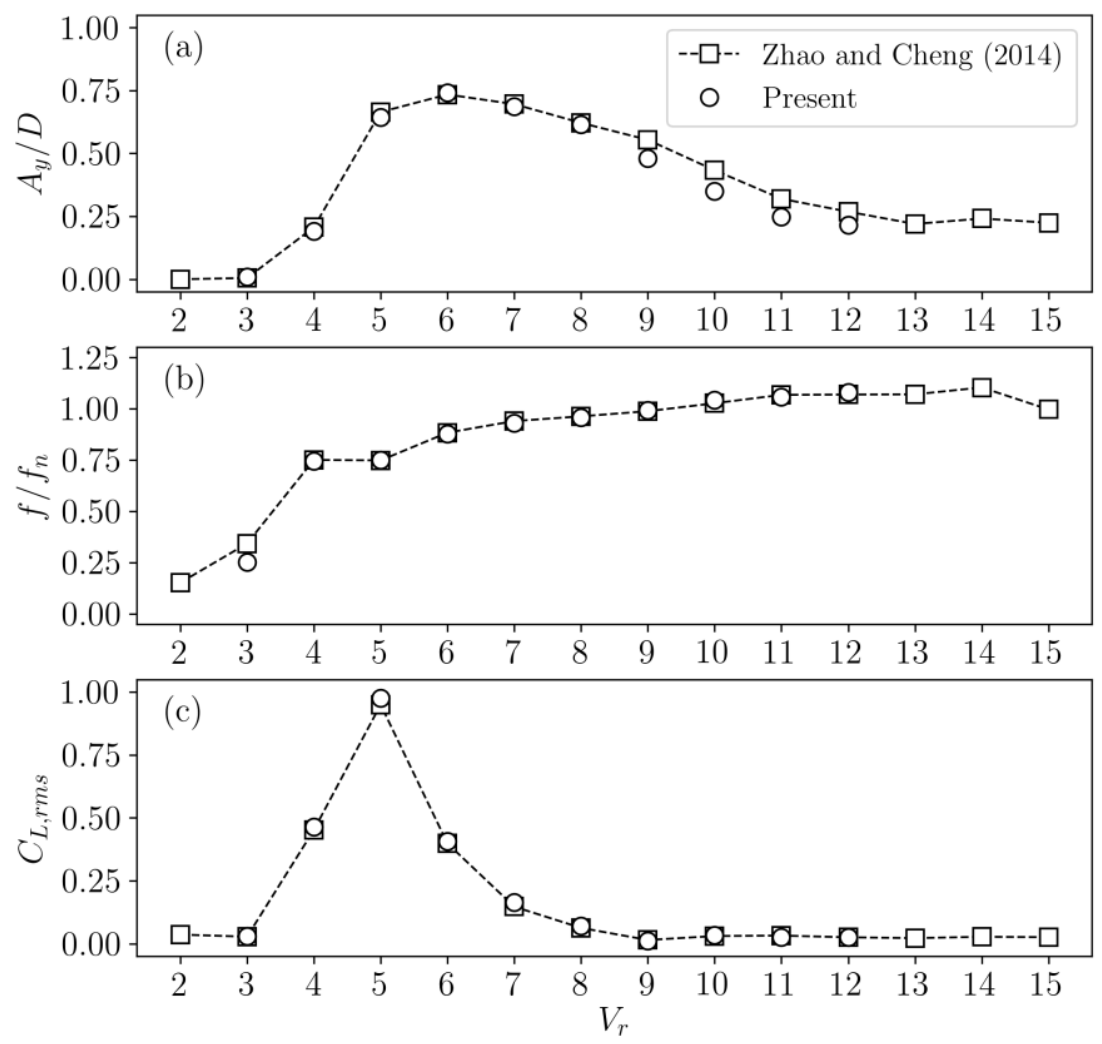

Figure 5 (a) Response amplitude, (b) response frequency and (c) lift coefficient of VIV of a circular cylinder of a finite length at $R e=300$ 


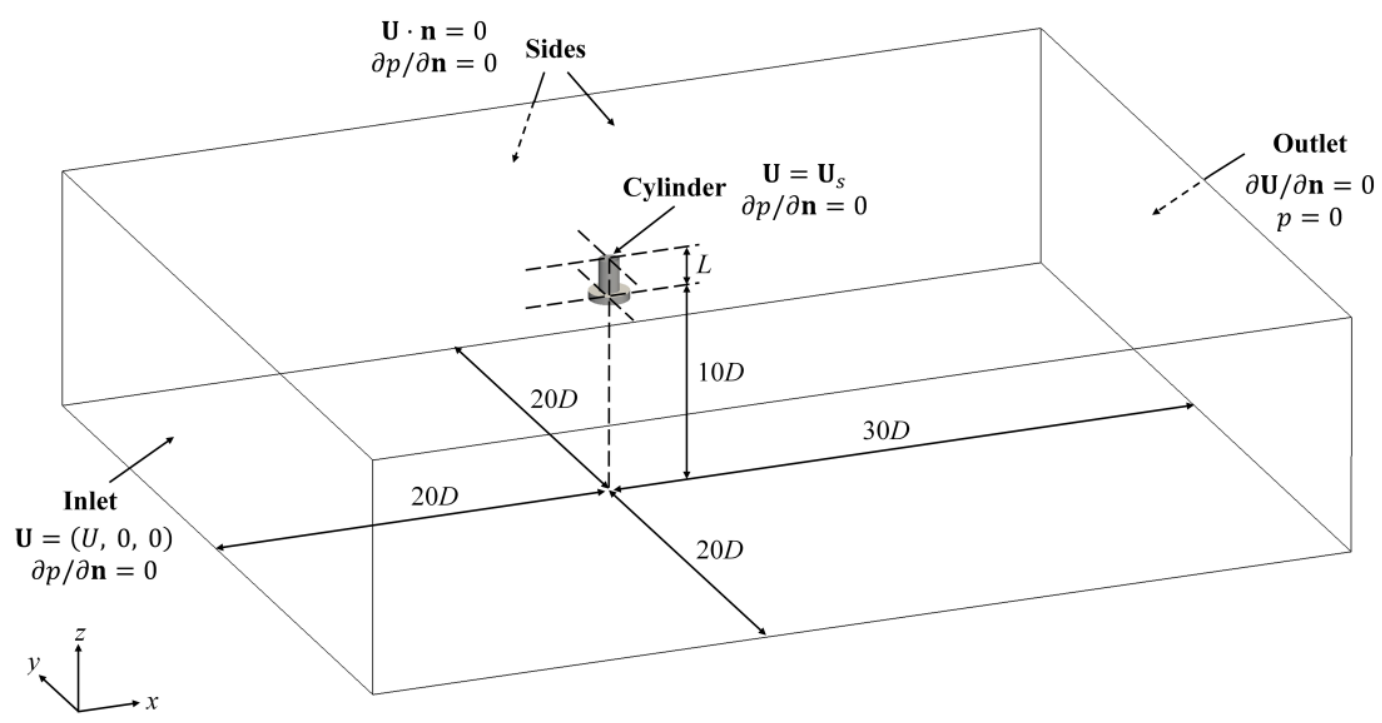

Figure 6 Dimensions and boundary conditions for VIV simulation of a circular cylinder with a base column: $L$ - overall length of cylinder; $D$-diameter of the upper part of cylinder

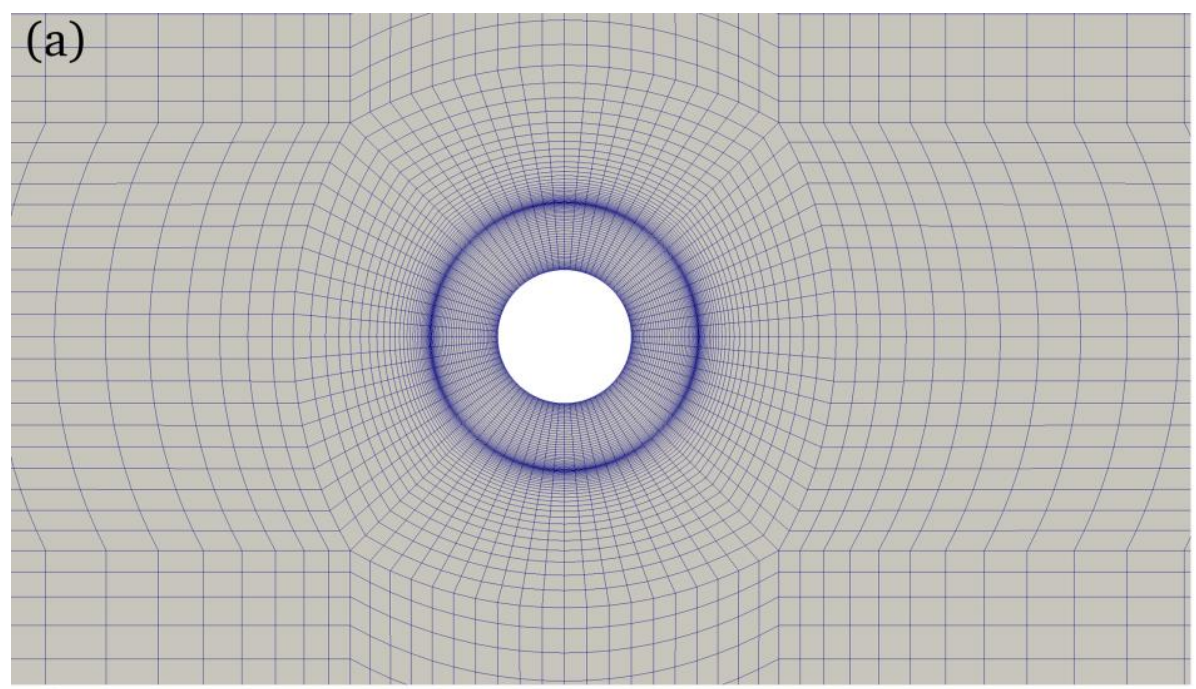

(b)

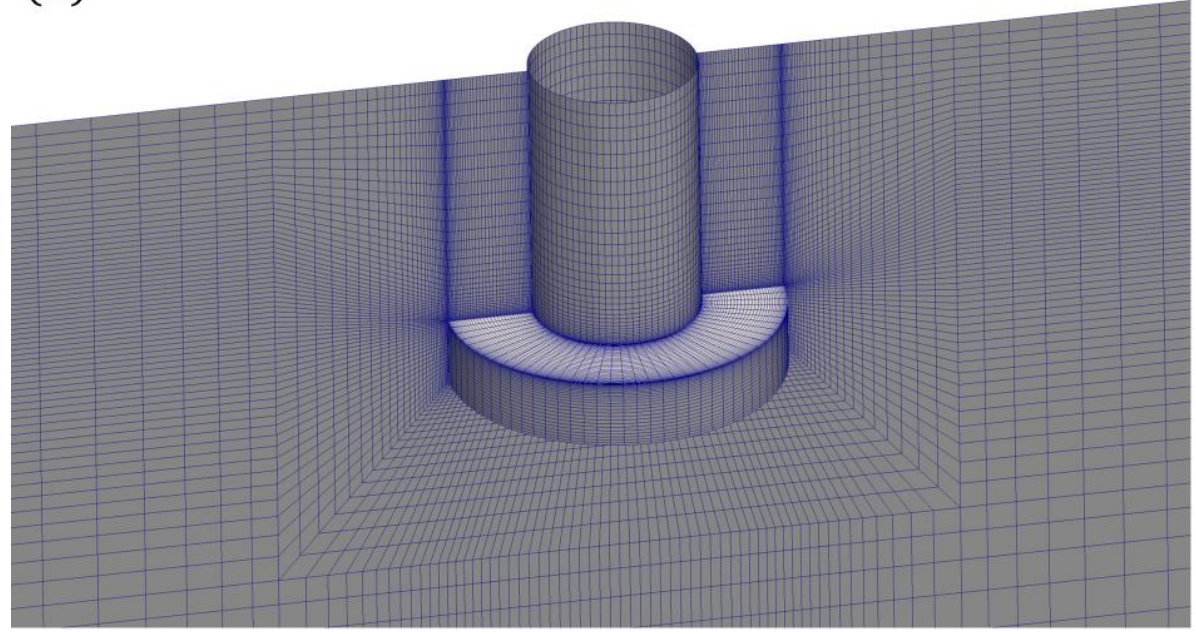

Figure 7 Computational mesh for VIV simulation of a circular cylinder with a base column: (a) top side; (b) cylinder surface 

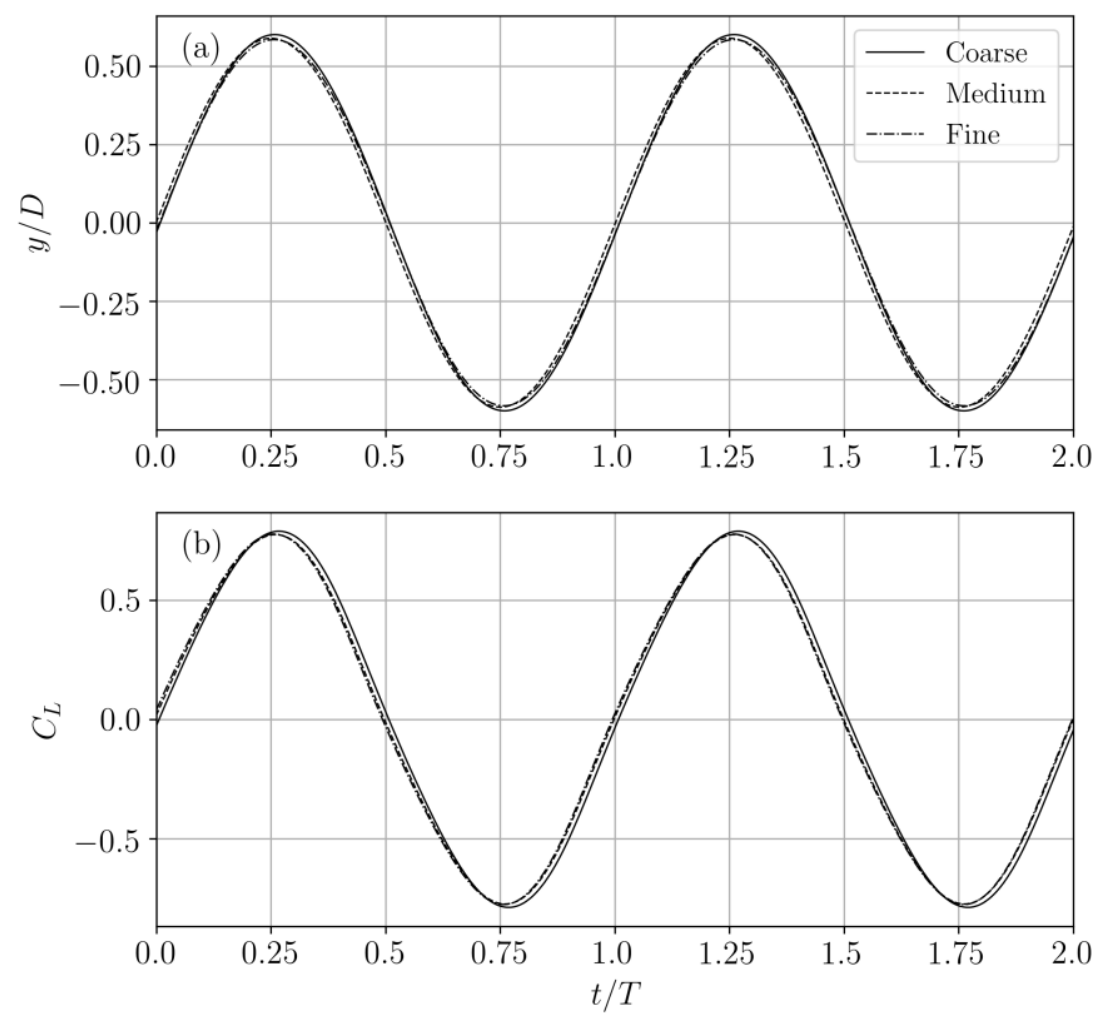

Figure 8 Comparison of time series of (a) response and (b) lift coefficient for VIV simulations of a cylinder with a base column at $V_{r}=6$ with different mesh density
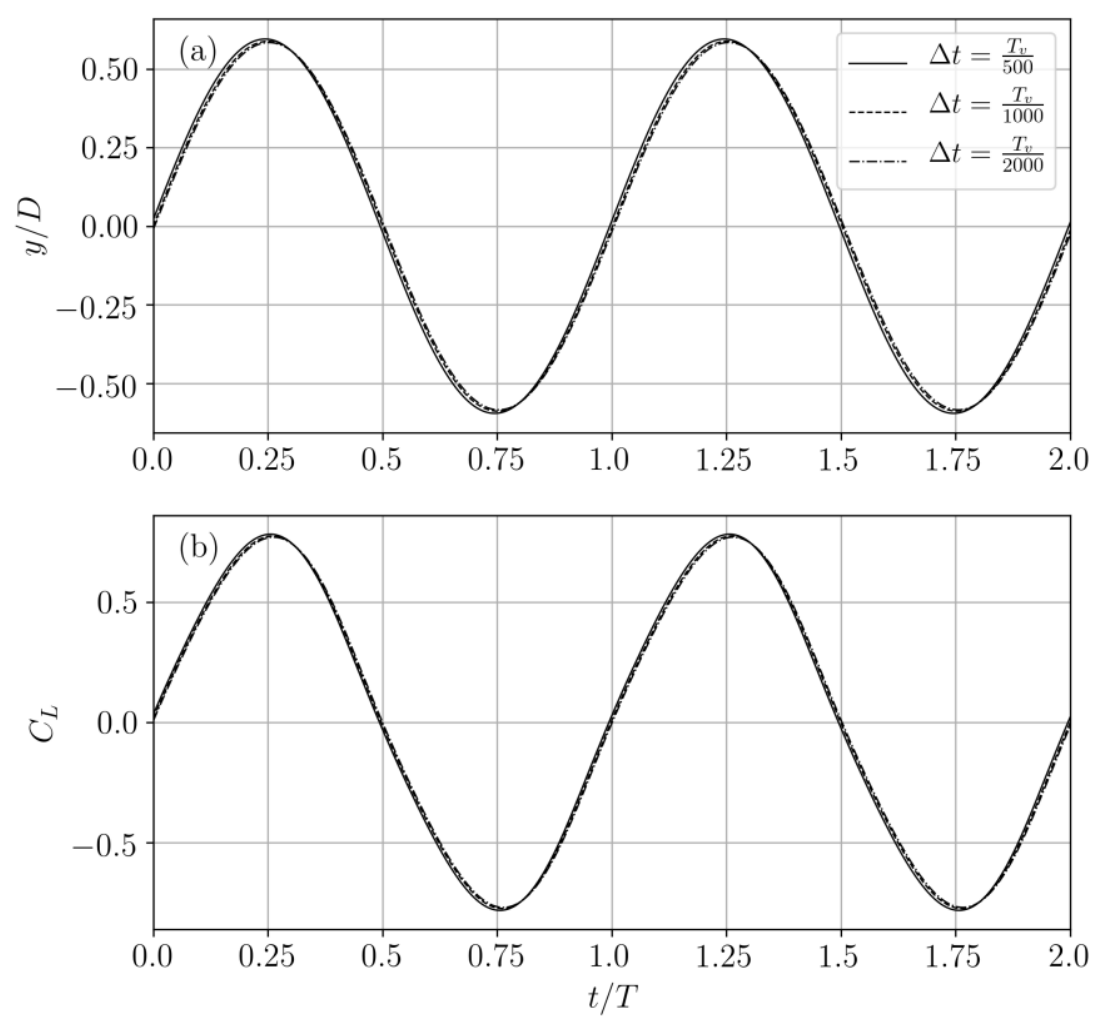

Figure 9 Comparison of time series of (a) response and (b) lift coefficient for VIV simulations of a cylinder with a base column at $V_{r}=6$ with different time-step size 


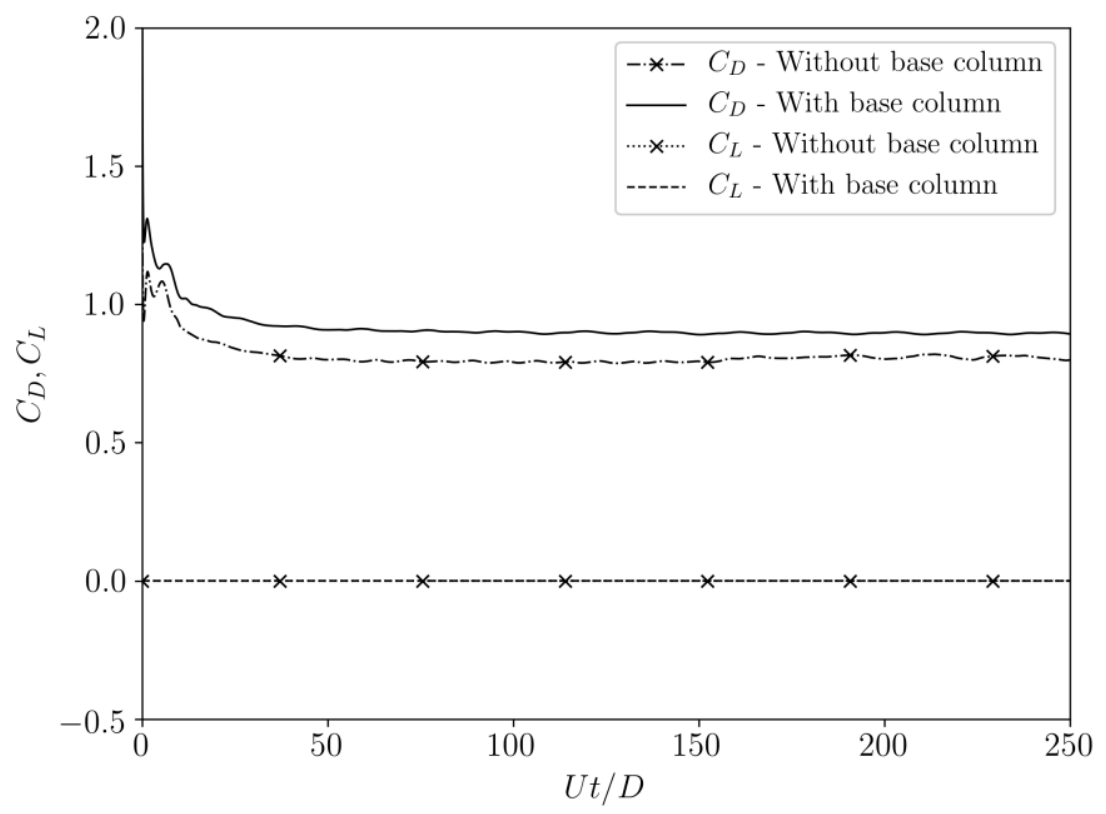

Figure 10 Time series of force coefficients of a stationary cylinder with and without a base column

(a)

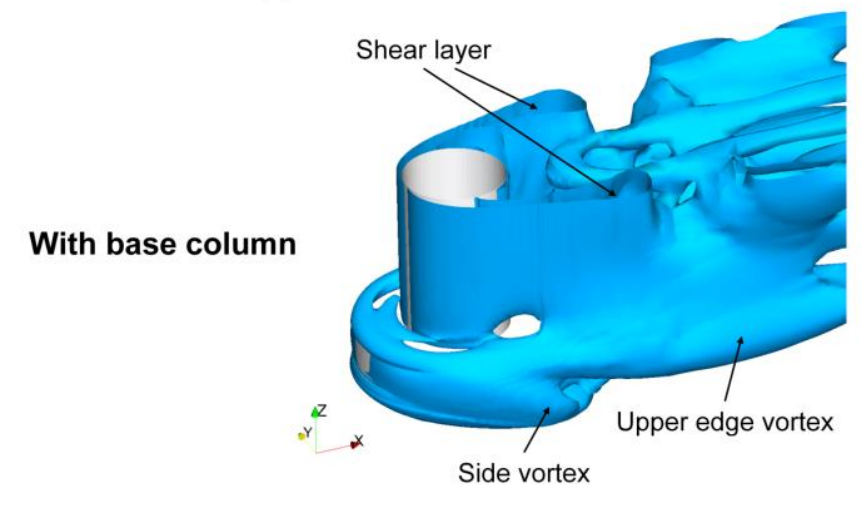

(c)

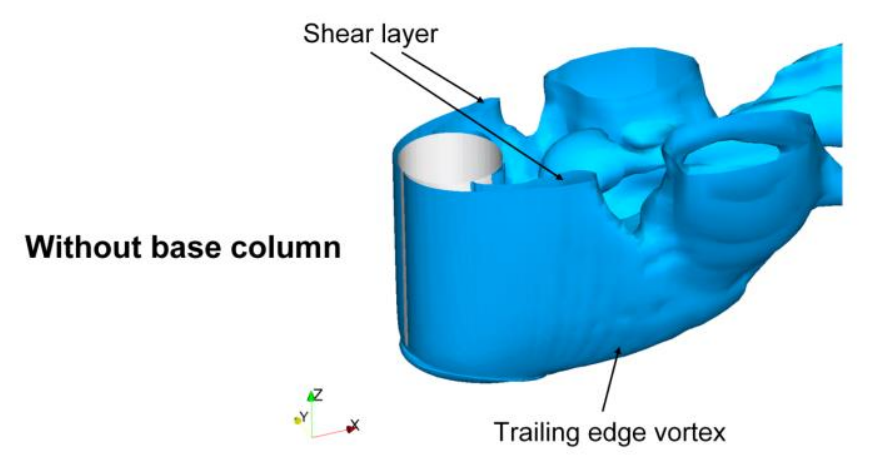

Top view (b)

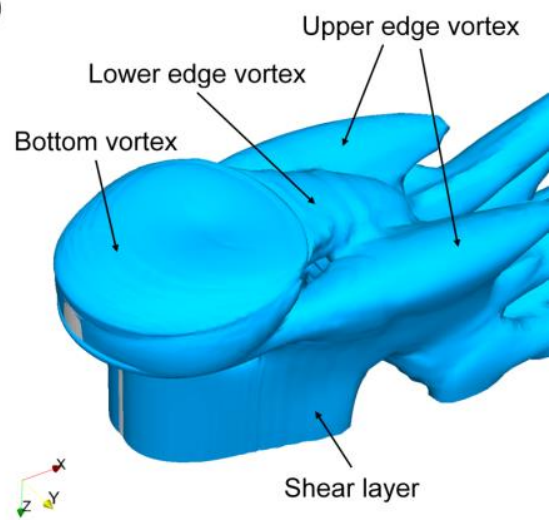

(d)

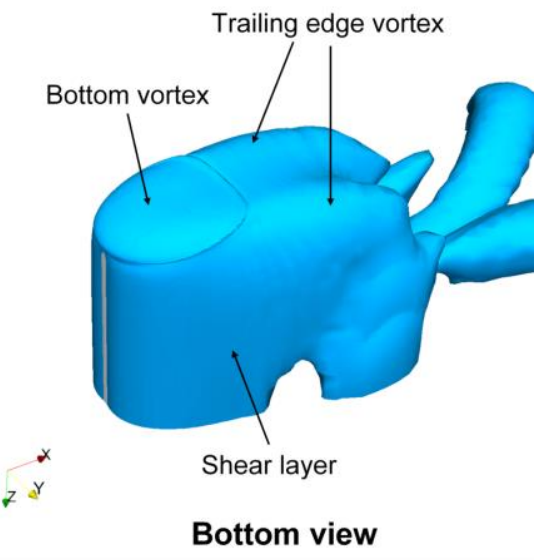

Figure 11 Vortex flow represented by iso-surface of $Q=0.2$ for a stationary cylinder with and without a base column at $R e=300$ 


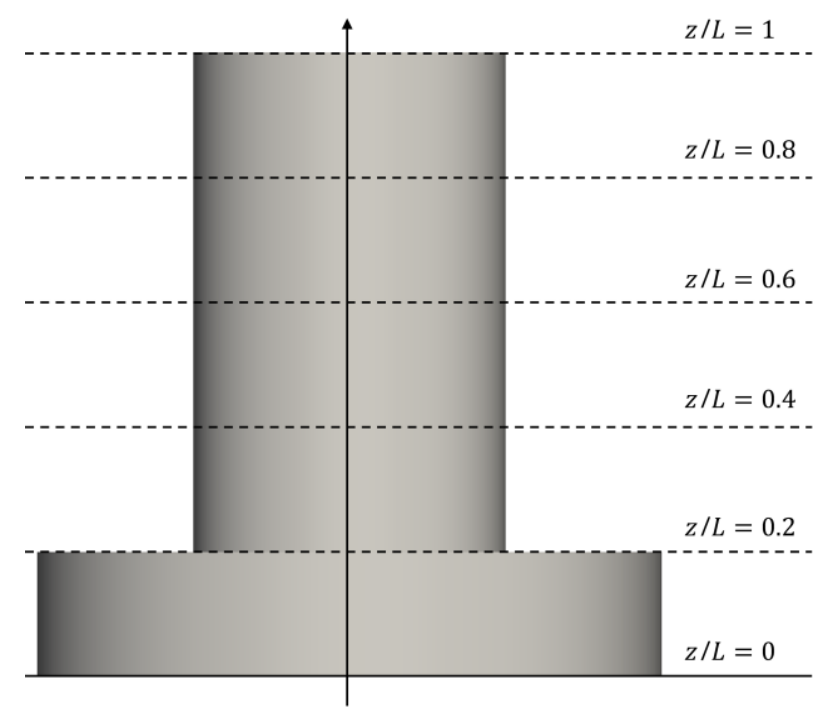

Figure 12 Schematic of slice planes for a cylinder with a base column
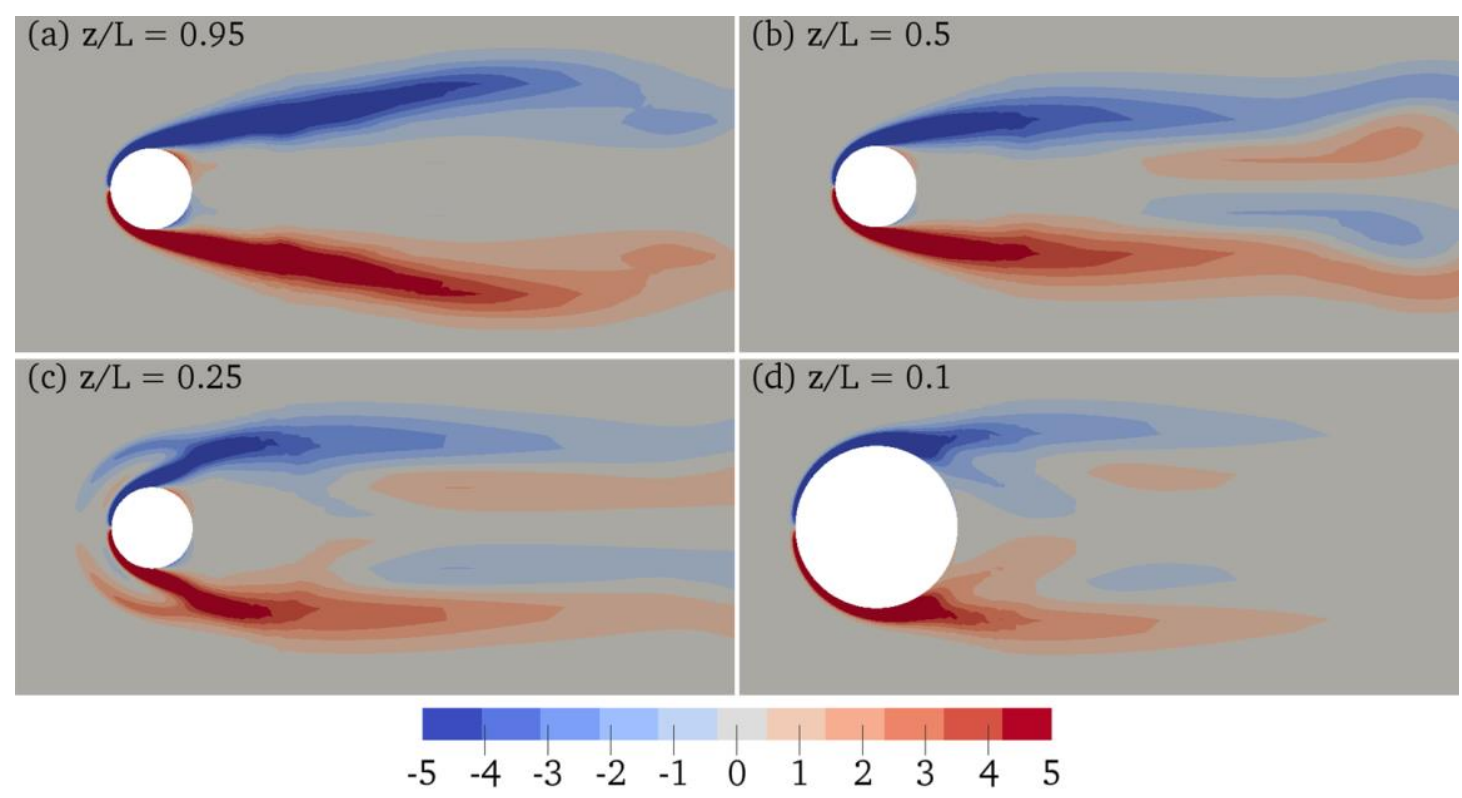

Figure 13 Contours of axial vorticity $\omega_{z}$ on four slice planes for a stationary cylinder with a base column at $R e=300$ 
(a) With base column

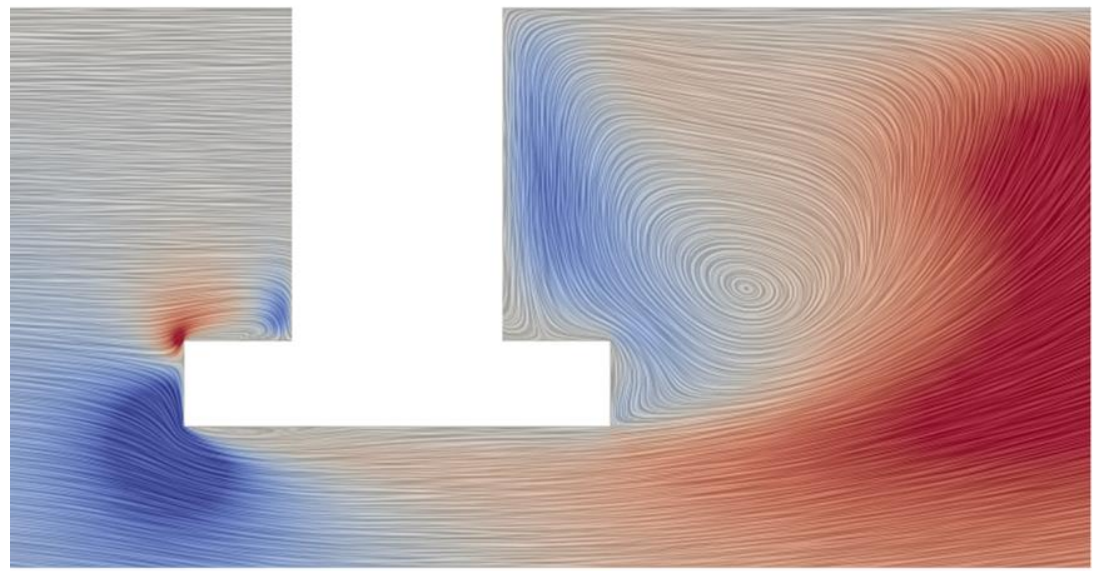

(b) Without base column
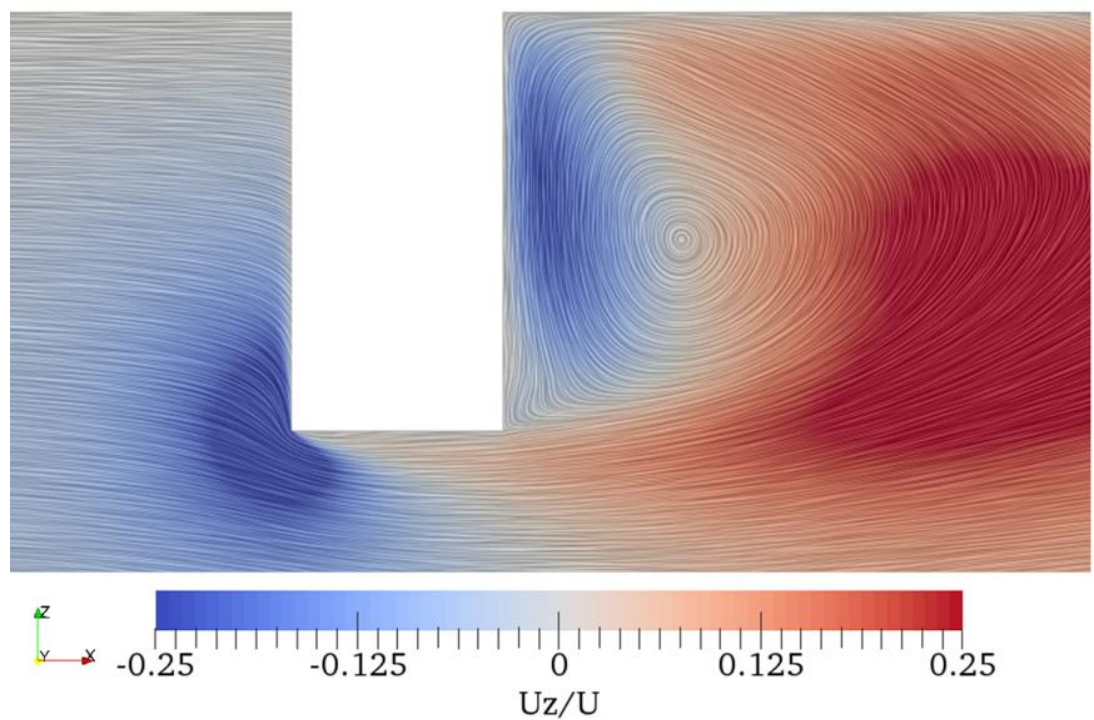

Figure 14 Comparison of streamlines coloured by non-dimensional vertical velocity at midplane $y=0$ between a stationary cylinder (a) with and (b) without a base column 

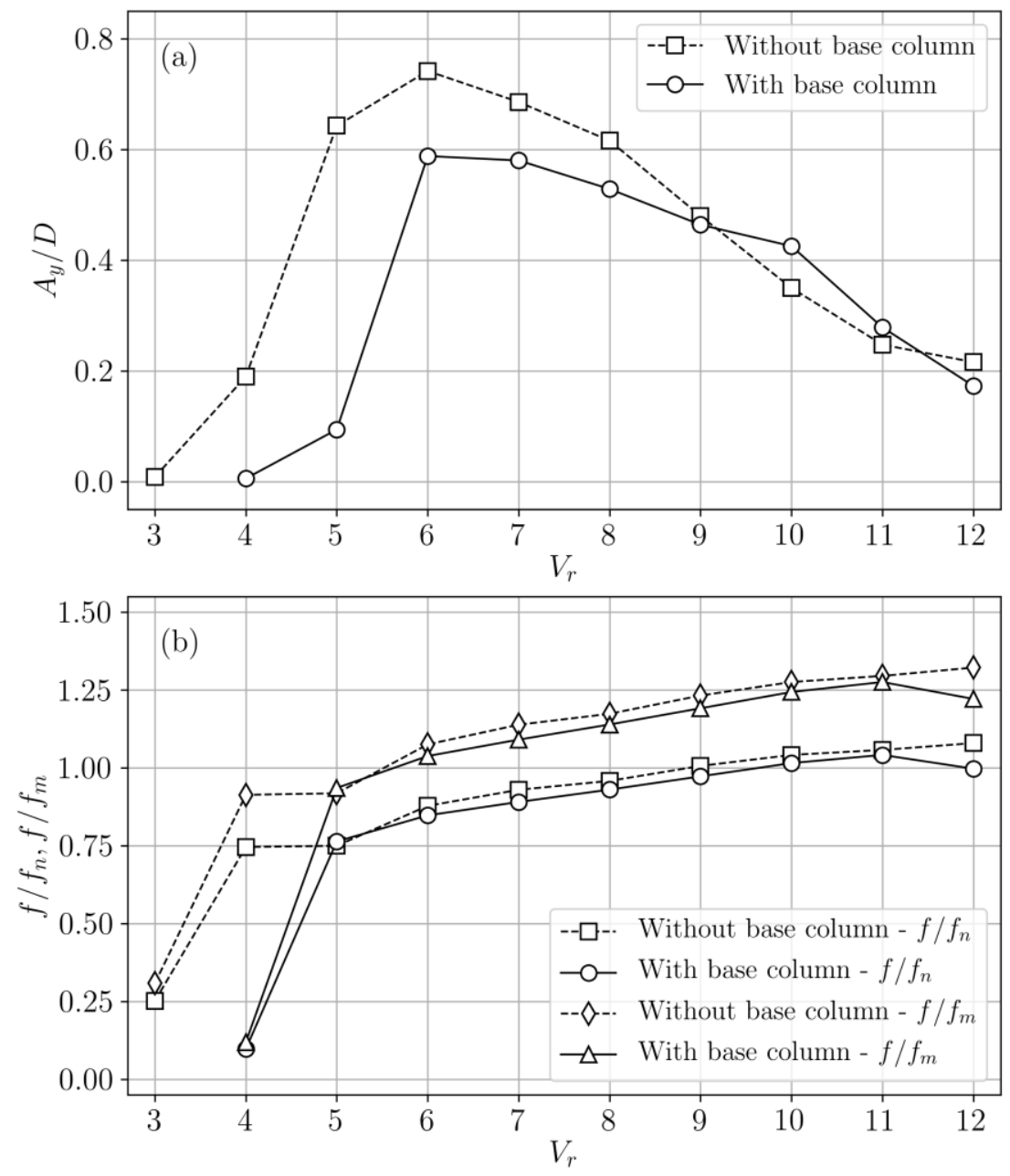

Figure 15 Comparison of (a) response amplitude and (b) response frequency against $V_{r}$ for VIV of a cylinder with and without a base column: $f_{n}$ - natural frequency of cylinder in vacuum; $f_{m}$ - natural frequency of cylinder in fluid 

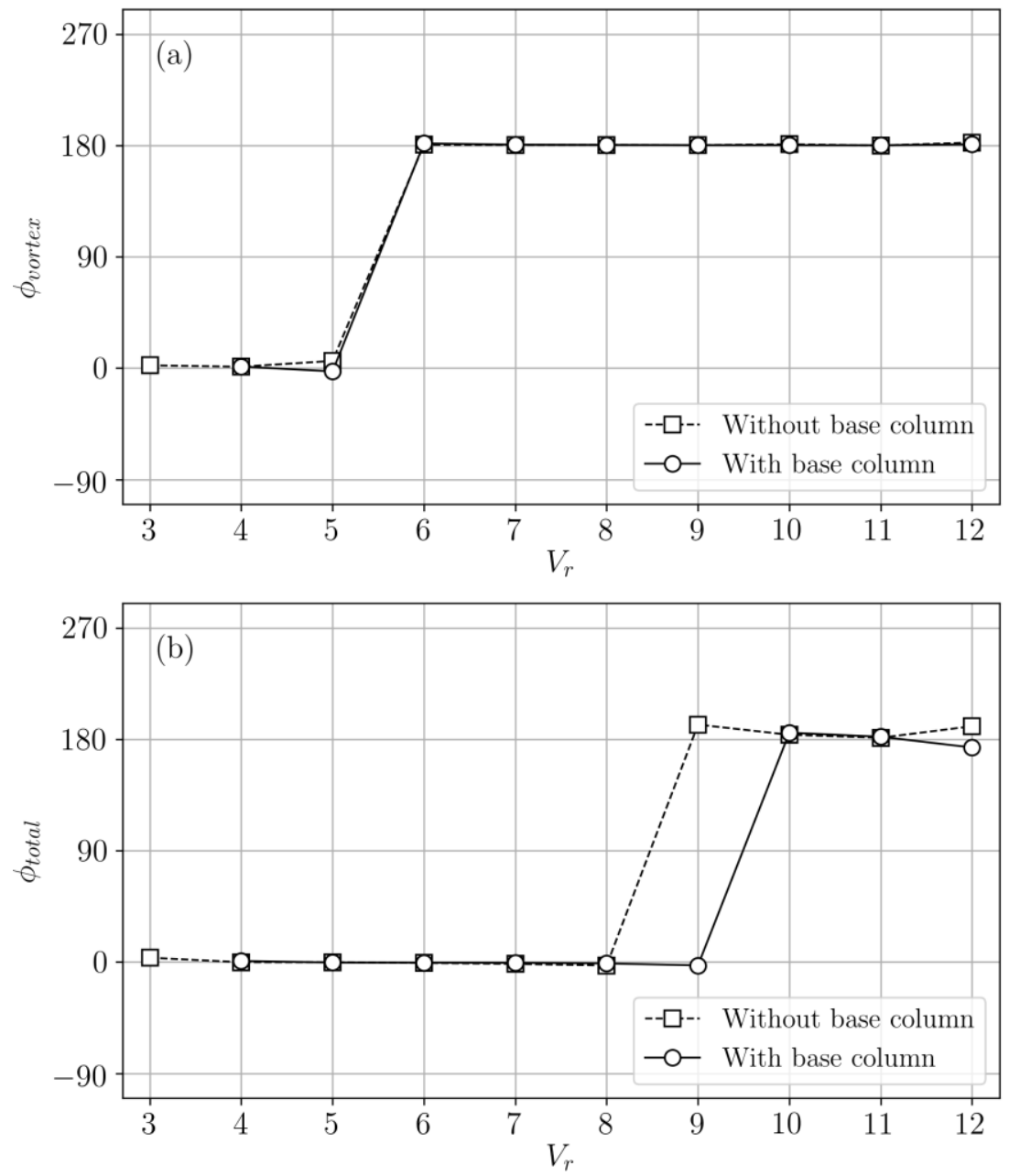

Figure 16 Comparison of (a) vortex phase and (b) total phase against $V_{r}$ for VIV of a cylinder with and without a base column 

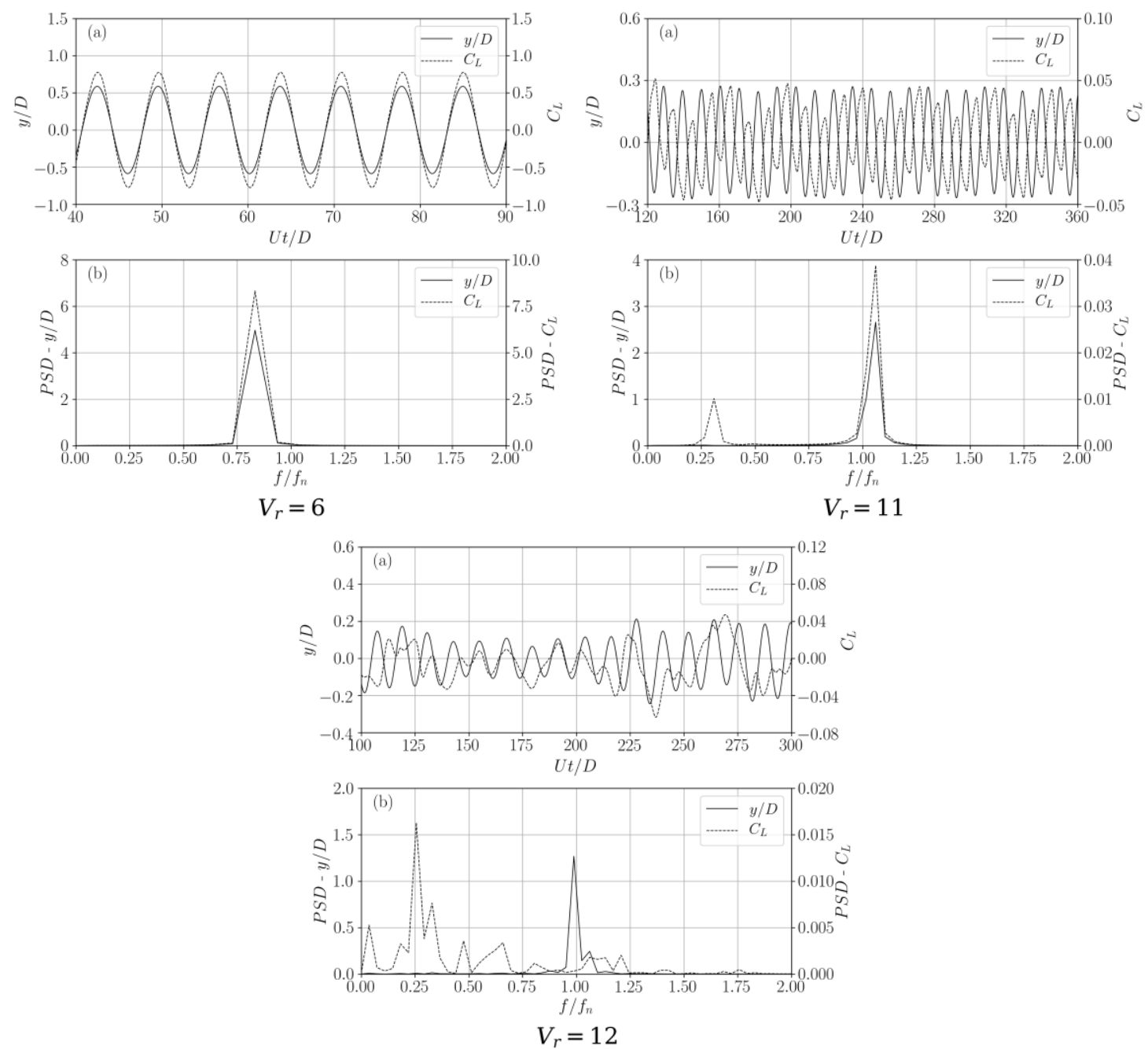

Figure 17 (a) Time series and (b) PSD of response and lift coefficient for VIV of a cylinder with a base column at various $V_{r}$
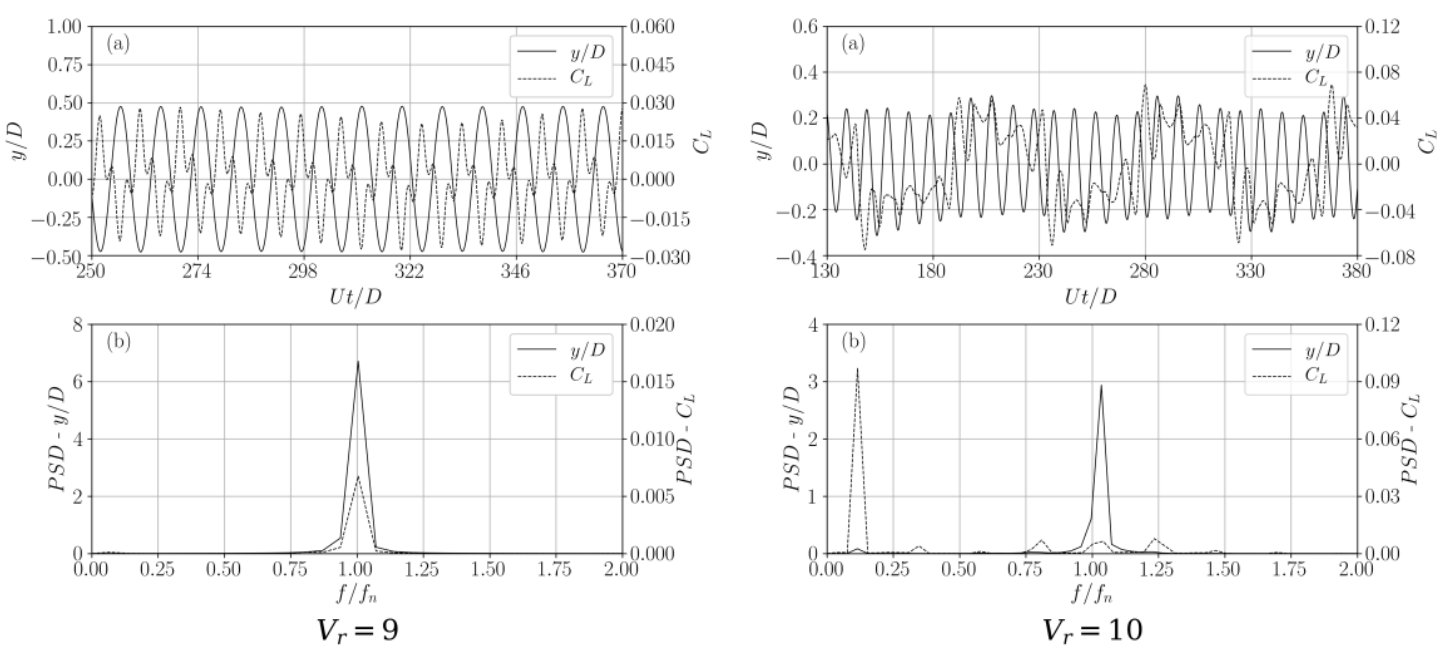

Figure 18 (a) Time series and (b) PSD of response and lift coefficient for VIV of a cylinder without a base column at various $V_{r}$ 


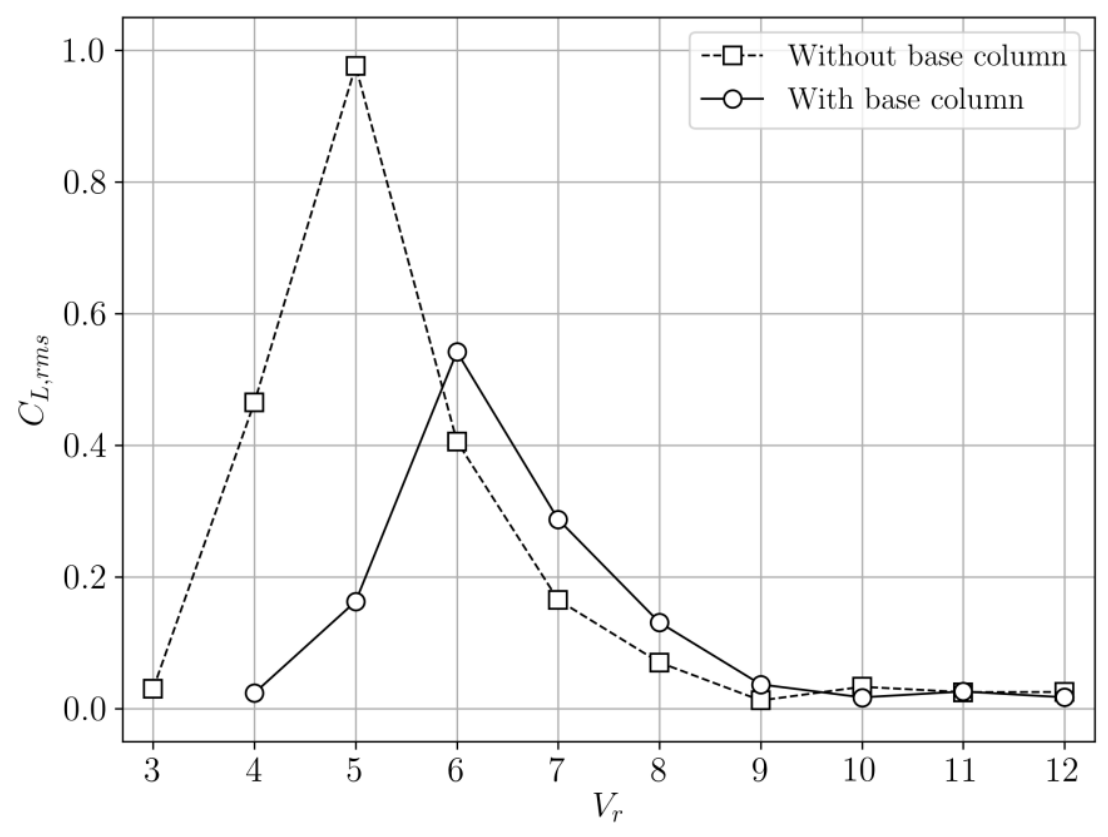

Figure 19 Comparison of lift coefficient against $V_{r}$ for VIV of a cylinder with and without a base column
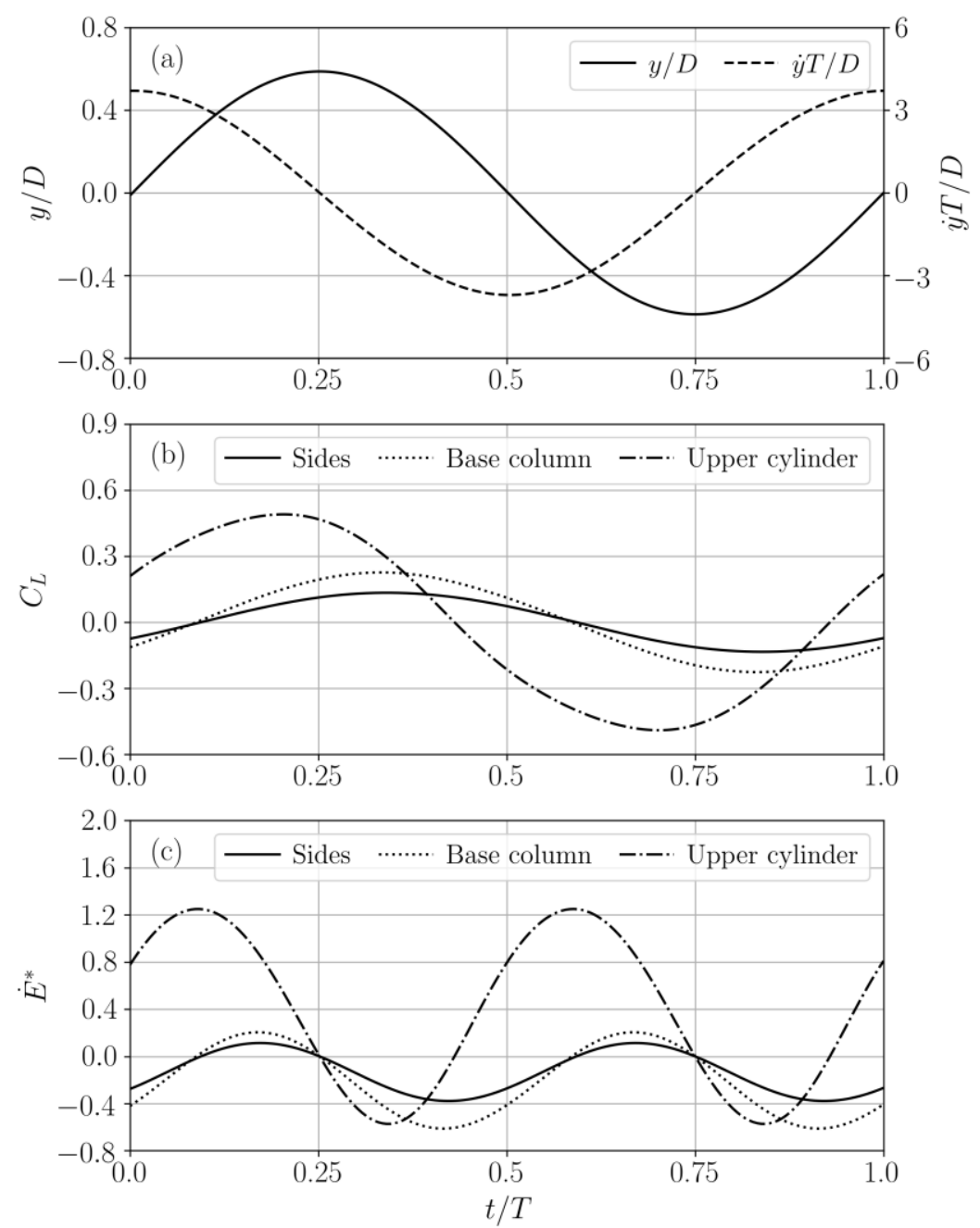

Figure 20 Time series of (a) response and velocity, (b) lift coefficient and (c) rate of energy transfer for VIV of a cylinder with a base column at $V_{r}=6$ 


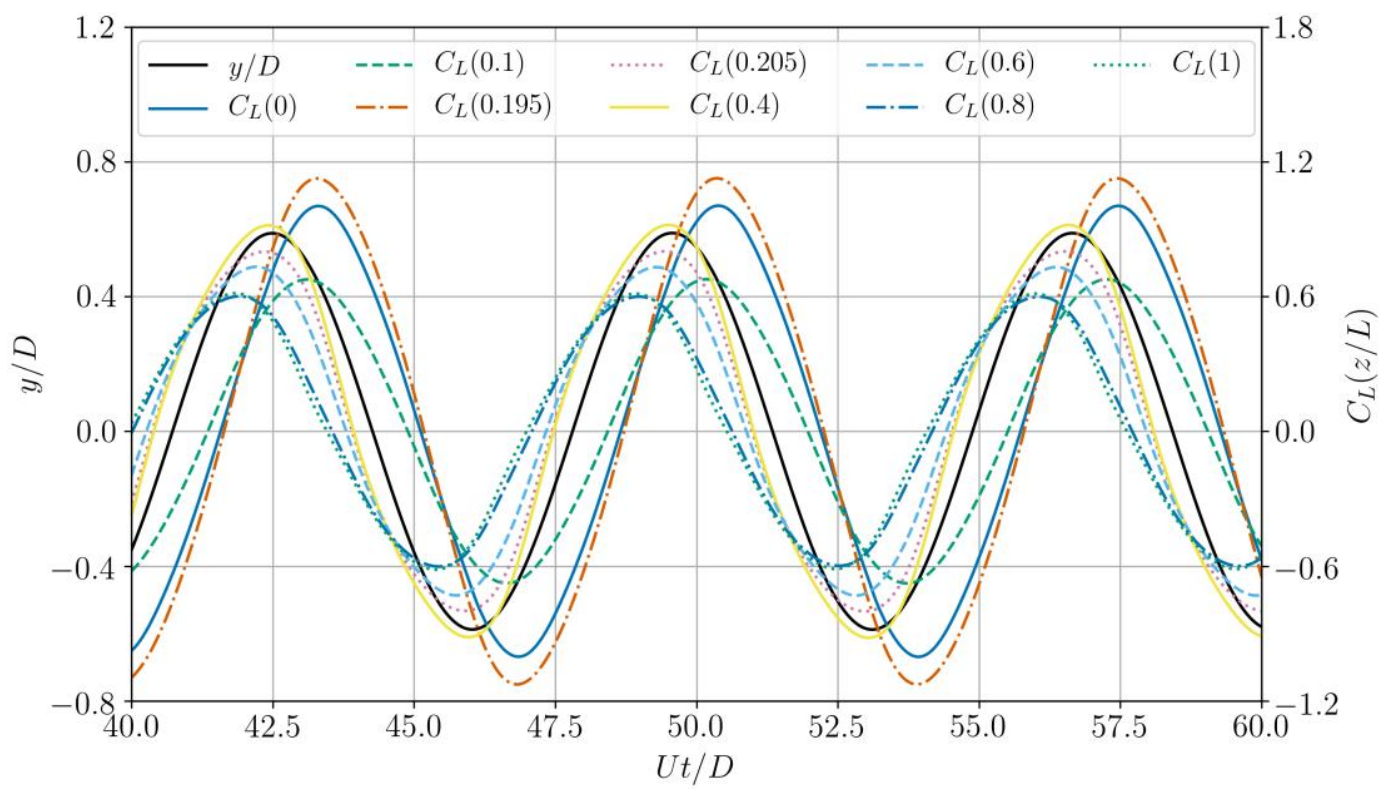

Figure 21 Time series of sectional lift coefficient at various $z / L$ for VIV of a cylinder with a base column at $V_{r}=6$
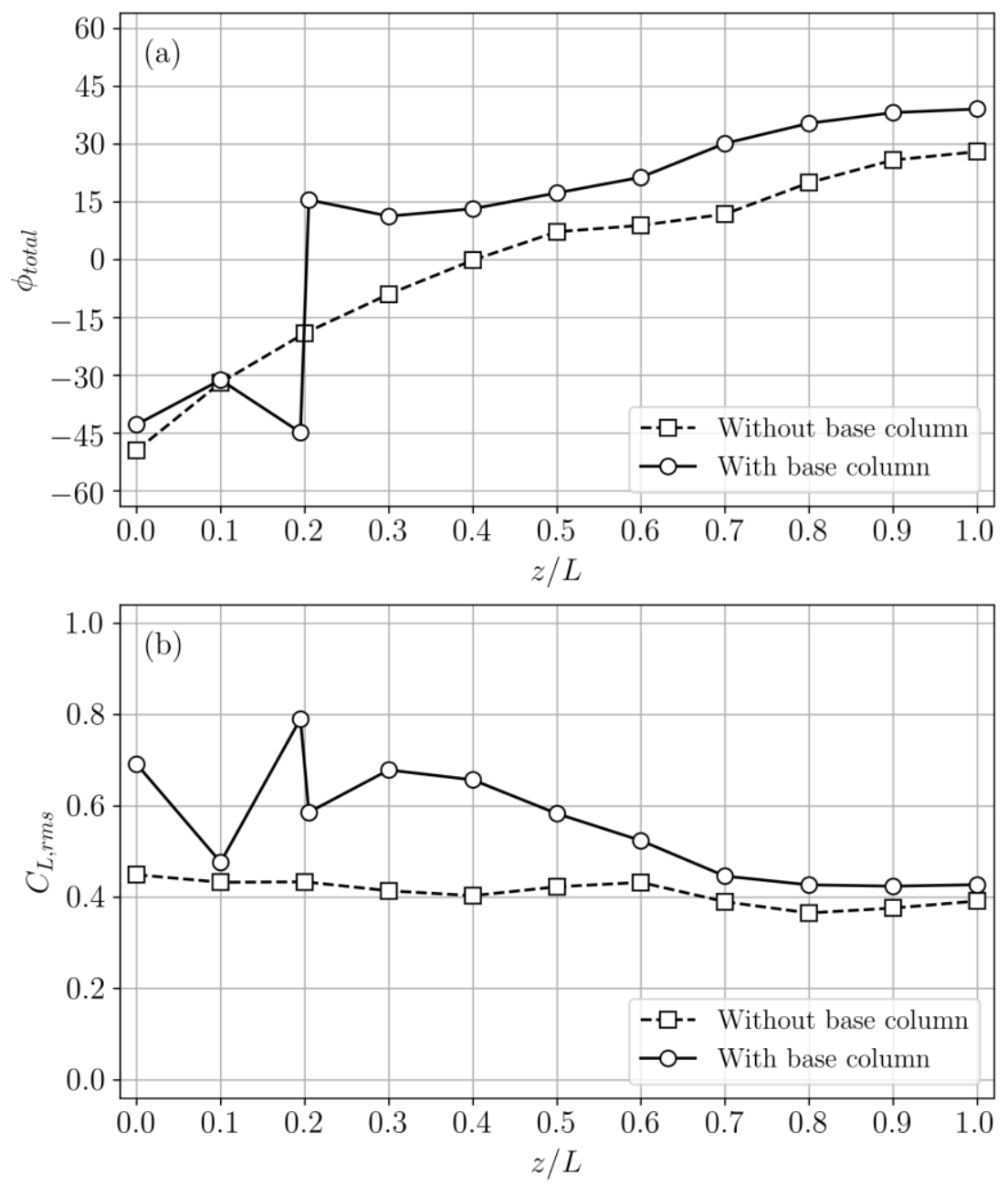

Figure 22 Comparison of (a) total phase and (b) sectional lift coefficient rms against $z / L$ for VIV of a cylinder with and without a base column at $V_{r}=6$ 
A

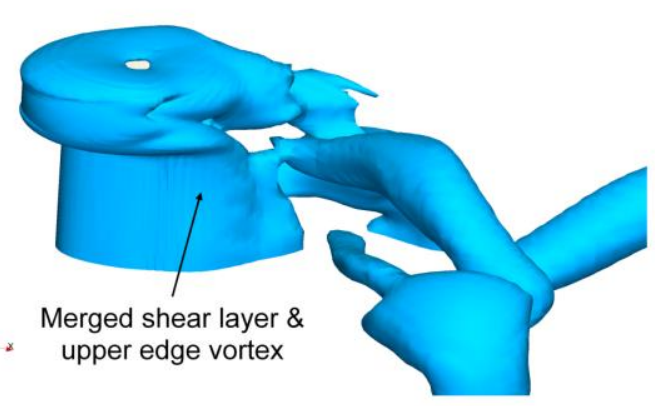

B

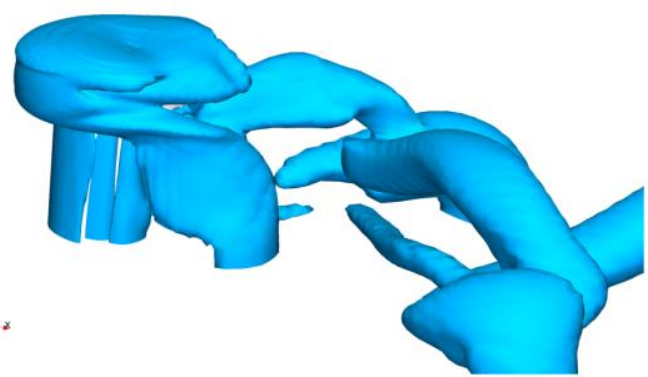

C

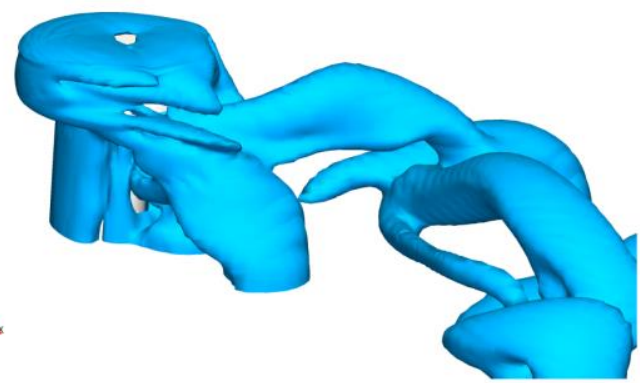

D

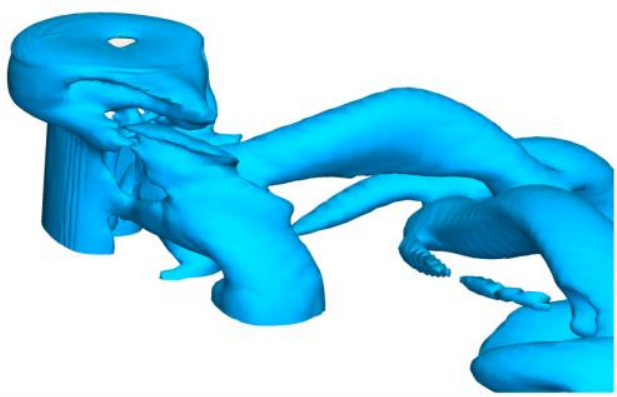

E

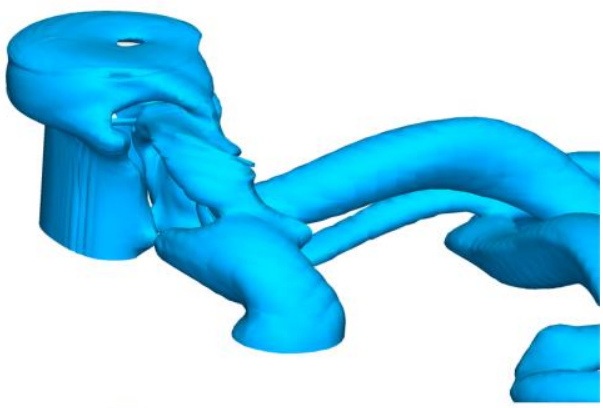

F

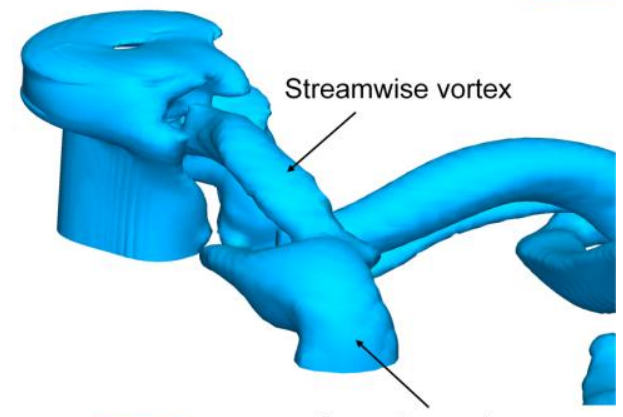

G
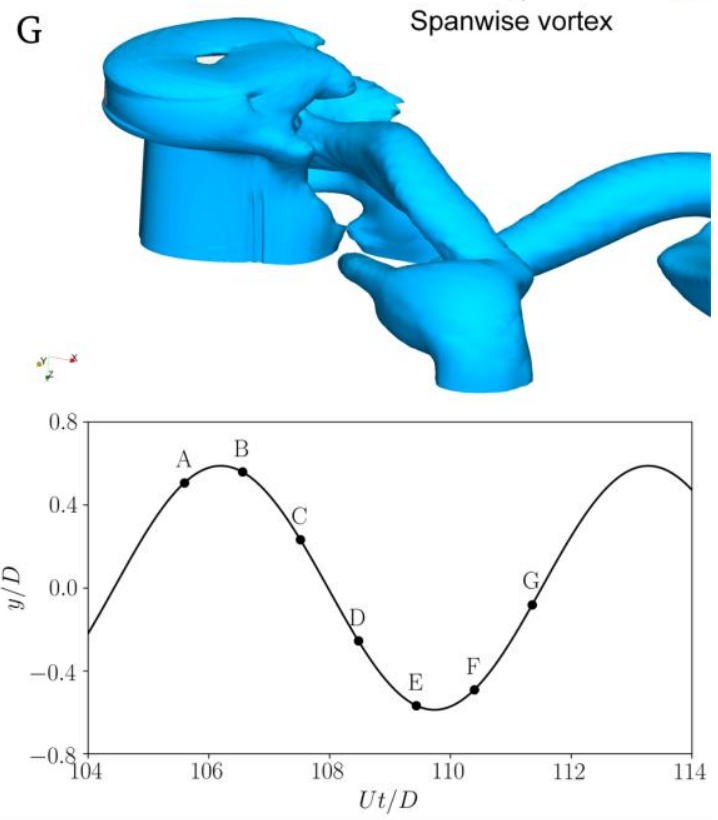

Figure 23 Vortex evolution represented by iso-surface of $Q=0.2$ for VIV of a cylinder with a base column at $V_{r}=6$ within one response cycle 


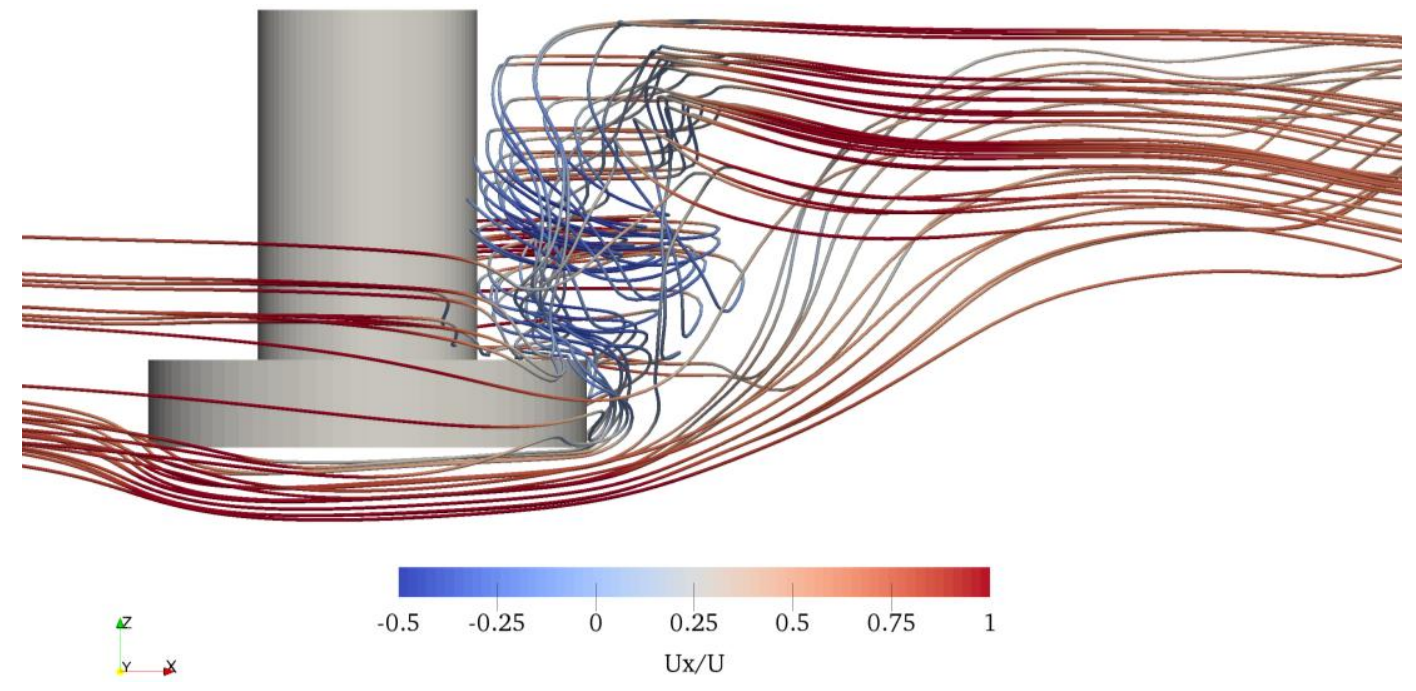

Figure 24 Streamline coloured by non-dimensional streamwise velocity for VIV of a cylinder with a base column at $V_{r}=6$

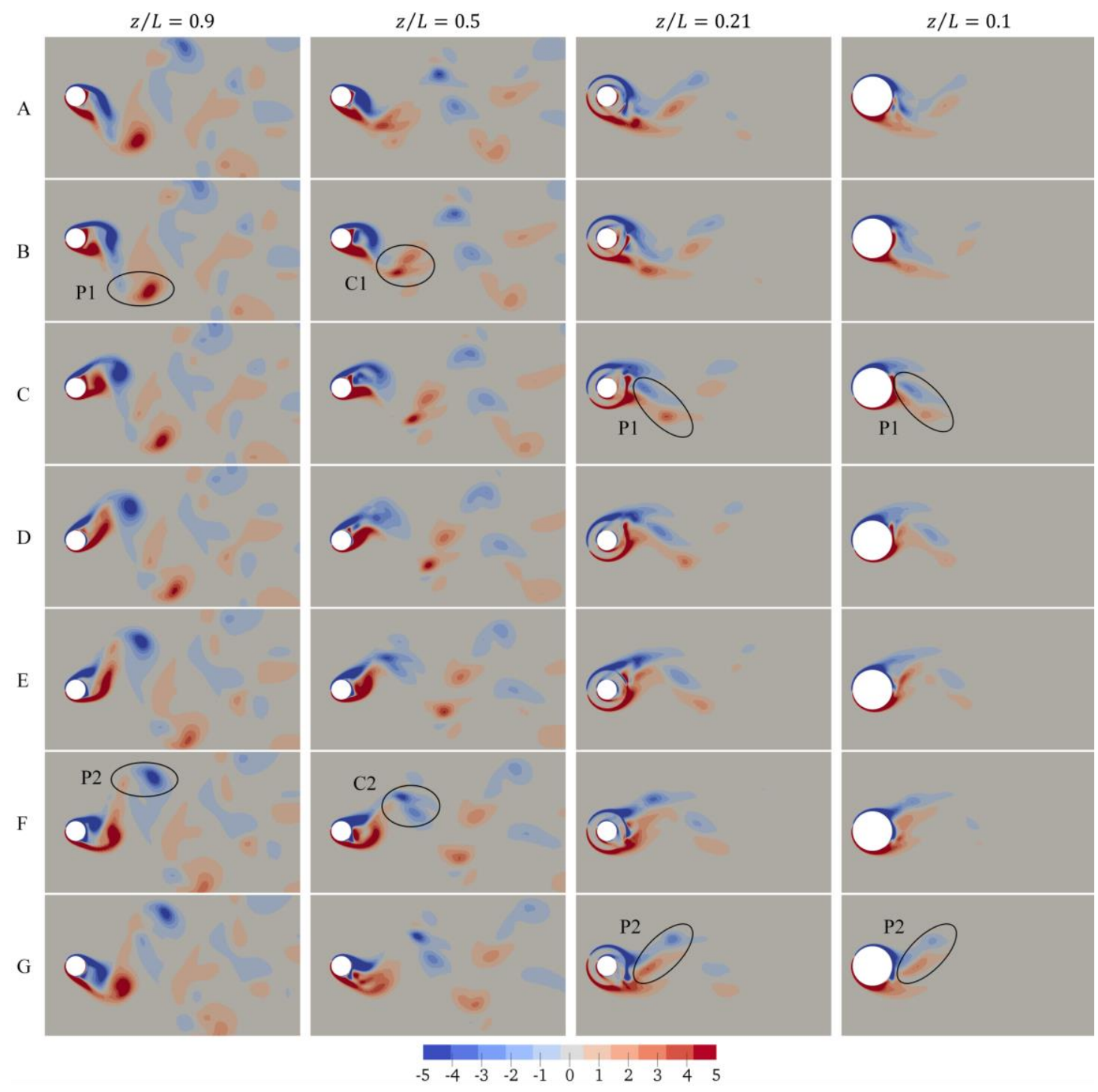

Figure 25 Contours of axial vorticity $\omega_{z}$ on four slice planes at different instants for VIV of a cylinder with a base column at $V_{r}=6$ 